\title{
X-Ray diffraction studies on electron density in organic crystals
}

\author{
P M Zorkii, A E Masunov \\ Lomonosov Moscow State University
}

ABSTRACT. The review presents the advances in precision $X$-ray diffraction studies on electron density in organic crystals carried out during the last several years. The methods for the elimination of and allowance for experimental errors, procedures used to interpret the results, and the characteristic features of the distributions of the deformation electron density in organic molecules are discussed.

The bibliography includes 126 references.

\section{Contents}

I. Introduction

II. Determination of the experimental distributions of $\rho$ and $\delta \rho$

III. Primary interpretation of electron density distributions

IV. Trends in the distributions of deformation electron density

\section{Introduction}

The spatial distribution of electron density $\rho(\mathbf{r})$ in a crystal was initially the principal result of X-ray diffraction analysis (XRDA). However, it was not until the middle of the 1960's that an increase in the experimental accuracy (automatic diffractometers), the correct allowance for systematic errors, the improvement of models (anisotropic thermal parameters), and the creation of special sets of programs made it possible to formulate the problem of the real physical significance of the asymmetry of the $\rho$ maxima.

The first X-ray diffraction studies on organic crystals, whose authors claimed to have achieved an objective description of the details of the electron density distribution $\rho(\mathbf{r})$, were published in $1966-1967 .^{1-5}$ This was associated with the use in Fourier synthesis of thermal parameters obtained from neutron diffraction data or data on the large angle scattering of X-rays. Thus the effects of the non-sphericity of the distribution of valence electrons were successfully separated from the effects of thermal motion. The redistribution of electron density on formation of chemical bonds was represented by the deformation electron density $\delta \rho(\mathbf{r})$ :

$$
\delta \rho(\mathbf{r})=\rho(\mathbf{r})-\rho_{0}(\mathbf{r})=\sum_{\mathbf{q}}(F(\mathbf{q})-\widetilde{F}(\mathbf{q})) \exp (\text { irq }),
$$

where $\rho_{0}$ is the electron density of the "precrystal" made up of spherically symmetrical non-interacting atoms, which are distributed and vibrate as in the crystal, $q$ the radius-vector of the inverse lattice site, $\widetilde{F}(q)$ the structural amplitudes of the "precrystal", and $F(q)$ the structural amplitudes of the crystal with the initial phases taken from $\tilde{F}(\mathbf{q})$.
Nevertheless, the procedures available to the investigators in the 1960's at best yielded a first aproximation to the solution of this fundamental problem. In subsequent years the improvement of various aspects of the method of determination of the functions $\rho$ and $\delta \rho$ made it possible to achieve reproducible (similar in analogous cases) results: $\delta \rho$ maxima of reasonable size were usually observed in the internuclear space of chemically bound atoms and it was usually possible to detect also peaks corresponding to lone electron pairs (LEP) and to discover the characteristic features of the distribution of $\delta \rho$ in the regions of hydrogen bonds.

The outcome of the studies of the charge distributions in organic crystals during the period from 1966 to 1984 was summarised in a review. ${ }^{6}$ In recent years, new steps of fundamental importance were taken in this research field. Here we have in mind (1) the improvement of the method of determination of experimental data, (2) progress in the interpretation of the distributions of $\rho$ obtained, and (3) the accumulation of information about the distributions of $\delta \rho$ for a wide variety of chemical compounds, which makes it possible to discuss successfully detailed features of chemical bonds. The aim of the present review is a consistent discussion of these three aspects of the latest studies on precision XRDA of organic crystals. The main outcome of the research under consideration apparently consists in the fact that, whereas previously mainly qualitative aspects of the subject were investigated, it has now become possible to guarantee the quantitative reliability of the details of charge distributions discussed.

\section{Determination of the experimental distributions of $\rho$ and $\delta \rho$}

The characteristic features of the precision X-ray diffraction experiment, the principles governing the construction of the distributions of $\rho$ and $\delta \rho$, and also the errors of these distributions have been examined in a review. ${ }^{7}$ Certain methods reducing the errors in the determination of experimental data and in their subsequent treatment and the latest examples of their application, demonstrating the great sensitivity of $\delta \rho$ maps to such correction, are presented below. The wide-scale use of the function $\delta \rho$ is due to two causes. Firstly, the difference between two convergent series (1) converges faster than either individual series and the termination of the series therefore affects $\delta \rho$ less than $\rho$. Secondly, the distribution of $\rho$ requires some kind of procedure for primary interpretation ${ }^{8}$ while the distribution of $\delta \rho$ is a result of one of the versions of such interpretation.

In order to obtain $\rho$, it is necessary in the first place to convert correctly the experimental intensities into the moduli of structural amplitudes, which involve the introduction of a series of corrections for systematic experimental errors. ${ }^{7}$ These are for example, the 
allowance for the anomalous and thermal diffuse scattering, extinction, and absorption.

Allowance for anomalous scattering is essential in the presence of heavy element atoms and is not usually carried out in their absence. Thus in the case of sulphur (thiourea) it causes little change in $\delta \rho .^{9}$ Allowance for thermal diffuse scattering is extremely important. ${ }^{10}$ Without it, the thermal parameters of atoms proved to be too low (for example, by $12 \%$ in oxalic acid), but this is not reflected in the qualitative features of the distributions of $\delta \rho$ in the region of chemical bonds and lone pairs. ${ }^{11}$

The greatest difficulty arises in allowing for the extinction contribution. Although at the present time methods are available for such allowance, the introduction of the appropriate corrections for single crystals of different size and shape leads to appreciable differences in the heights and positions of the $\delta \rho$ maxima. ${ }^{12}$ The intensity and small wavelength of synchrotron radiation make it possible to investigate single crystals whose diameter is less than $0.1 \mathrm{~mm}$. The small size of the crystals diminishes the influence of extinction and absorption. ${ }^{13}$ These and other advantages of synchrotron radiation (the possibility of reducing anomalous scattering by modifying the wavelength, the increased accuracy of the analysis of the peak profile by virtue of the small divergence of the beam) make its use in precision X-ray diffraction analysis extremely promising.

Appreciable changes in $\delta \rho$ maps (especially near the nuclei) occur even after small changes in the scaling factor and it is therefore refined separately with respect to the entire set of structural amplitudes, with the remaining variable parameters fixed. Recently an alternative procedure has also been used-the determination of the scaling factor from hard $\gamma$-ray diffraction data ${ }^{14}$ or from neutron diffraction data. ${ }^{15}$

As already stated, the distribution of $\delta \rho$ is less subject to the influence of the termination of the series than the distribution of $\rho$, but in the case of $\delta \rho$ it is more difficult to apply the corresponding correction. There is a prospect that in the solution of this problem (both for $\rho$ and $\delta \rho$ ) it will be possible to abandon the traditional Fourier method, having replaced it by the entropy maximisation method. The latter makes it possible also to reduce the role of random errors, which increases the resolution of the electron density maps. ${ }^{16,17}$ The essential feature of the method of Kuz'min et al. ${ }^{18}$ consists in the fact that the coefficients of the series, corresponding to weak reflections and assumed to be zero in the usual XRDA scheme, are found from the conditions

$$
S=-\int \frac{\rho(\mathbf{r})}{F(000)} \ln \frac{\rho(\mathbf{r})}{F(000)} d \mathbf{V} \rightarrow \max
$$

Here the function $\rho(r)$ is regarded as the distribution of the probability density, the best distributions being selected from all the distributions agreeing with the available information. The application of this procedure requires, however, a large amount of computer time. ${ }^{16}$

Hitherto we have spoken of the improvement of the determination of the moduli of structural amplitudes. Another possibility of refining the distributions of $\rho$ obtained from a diffraction experiment consists in improving the calculation of the initial phases. In the standard XRDA, they are calculated in the anisotropic harmonic approximation of spherically scattering atoms. In order to obtain accurate distributions of $\rho$, use is made of better models: the $L$-shell method (the cleaved atom method), the $\kappa$-method, the two-centre models of charge clouds on bonds and lone pairs (Stewart, Hellner), and different versions of the multipolar models (Coppens, Hirshfeld,
Stewart, Tsirel'son). All these methods, which are mentioned in order of increasing complexity, have been described in a review. The importance of allowing for the anharmonicity of thermal vibrations has been confirmed ${ }^{19}$ in relation to $\gamma$-aminobutanoic acid. The methods listed have been compared in two communications. ${ }^{20,21}$

The most complex (and because of this the most flexible) of the models enumerated contain a large number of parameters, which sometimes reaches the number of the accessible independent structural amplitudes. The problem of eliminating the correlations between these parameters has therefore arisen. Here the use of neutron diffraction and spectroscopic data as well as the superposition of additional physical conditions, in particular the determination of the relation between the distribution of $\rho$ and the one-determinant wave function of the crystal, are helpful. One of the new examples of this approach has been provided by Frichberg. ${ }^{22}$

A relatively new step has been the use of experimentally measured initial phases. The anomalous scattering effect can be used for this purpose. In recent years, such studies have been carried out. In the presence of heavy atoms, anomalous scattering can be optimised by adjusting the wavelength of the synchrotron radiation. ${ }^{23}$ It has also been suggested ${ }^{24}$ that the initial phases be determined from intensity measurements at two wavelengths close to the characteristic wavelength, the wavelength of the $\mathrm{X}$-ray generated by the electron beam directly in the crystal investigated being determined by the angle of incidence of the electrons relative to the modal plane. This method is applicable also to substances without heavy atoms, for example, to oxygen-containing organic compounds.

The method based on analysis of three-wave diffraction profiles under the conditions of the Renninger effect (interference occurring when two series of planes assume simultaneously the reflecting position) has been applied in practice. ${ }^{12,25,26}$ This method makes it possible to determine experimentally the initial phases of certain reflections. A precision study of the $\mathrm{Si}$ crystal (involving measurement of the intensities and determination of the initial phases of the "forbidden" reflections) made it possible to increase by an order of magnitude the accuracy of the determination of $\delta \rho$ [mean square error $\sigma(\delta \rho) \simeq 0.005{\mathrm{e} \AA^{-3} \text { ] }}^{25}$ and to discover on the pseudostatistical $\delta \rho$ distribution map a fine effect involving the splitting of the peak on the $\mathrm{Si}-\mathrm{Si}$ bond into two components. ${ }^{26}$

The experimental determination of the initial phases on the basis of three-wave diffraction apparently represents the most promising aspect of studies of this kind. However, despite the clear advantages of the direct experimental methods for the determination of the initial phases listed above, they still remain too complex to hope for their rapid and extensive application on a large scale.

\section{Primary interpretation of electron density distributions}

The distribution of the total electron density contains in principle exhaustive information about the nature of intramolecular and intermolecular interactions ${ }^{10}$ (and also about all the one-electron properties of the system ${ }^{7}$ ), but its direct interpretation is difficult. ${ }^{8}$ To facilitate the use of such information, additional procedures and concepts are introduced. They will be examined in the present Section (in relation to examples taken from studies in recent years).

The simplest numerical characteristics of continuous distributions of $\rho$ are the effective point moments, in particular the atomic and bond charges. They can be obtained not only at the refinement 
stage (in the model of multipolar pseudoatoms or charge clouds) but also subsequently from the $\delta \rho$ distribution constructed. For example, it has been proposed ${ }^{27}$ that $\delta \rho$ peaks be fitted by ellipsoids of rotation and that the bond charge be calculated by integration with respect to the entire volume of the ellipsoid. Different procedures for the separation of $\delta \rho$ into contributions corresponding to individual atoms have been compared in another communication. $^{28}$ Atomic charges are useful in the calculation of the properties of the crystal (for example, in the calculation of the electrostatic field gradients at nuclei ${ }^{7}$ or of the Coulombic components of the intermolecular interaction ${ }^{6}$ ) and in the analysis of the "electronic structure" of molecules. Thus the employment of pseudoatomic charges, determined from diffraction data, made it possible to detect the inductive effect in molecules of barbital ${ }^{29}$ and tetrafluoroterephthalodinitrile ${ }^{30}$ and an unusual methylene hydrogen bond in $\gamma$-aminobutanoic acid: ${ }^{9}$

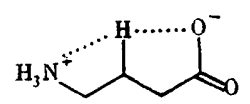

In order to weaken the dependence of the effective atomic charge on the method used to subdivide the continuous distribution, it has recently been suggested ${ }^{31}$ that the charge of a "local region", defined as the sum of the effective charges on a given atom and on the neighbouring atoms linked directly to it, be brought into correspondence with each atom. This approach yields more "reasonable" values. For example, the carbon atoms of an aromatic nucleus in the naloxone ion $\mathrm{C}_{19} \mathrm{H}_{22} \mathrm{NO}_{4}^{+}$have been found to carry a larger negative charge than the aliphatic carbon atoms, the nearest $\mathrm{N}$ and $\mathrm{O}$ atoms carrying the greatest negative charge.

Pseudoatomic moments can also be used to bring the parametric description of $\rho$ closer to orbital ideas. ${ }^{32}$ The calculation of the electron populations of the $d$-orbitals of $\mathrm{Fe}^{2+}$ in the porphyrin complex with the aid of a multipolar model yielded values close to those expected for the high-spin ${ }^{5} F_{2 g}$ configuration. The observed deviations indicate the presence of dative bonds with the ligands. ${ }^{33}$ A more general approach consists in the application of the valence form-factors, i.e. the atomic scattering factors, taking into account the valence state and not averaged with respect to orientations: this approach also yields $p$-orbital populations. ${ }^{34}$

Another way of interpreting the distribution of $\rho$ in chemical terms involves the density matrix method. ${ }^{7}$ In this method the calculation of the elements of the bond charge-order matrix elements involves adjustment to $\mathrm{X}$-ray diffraction data. An unambiguous calculation is achieved by imposing an additional condition involving the minimisation of the energy functional. ${ }^{35}$ However, the finite nature of the basis set makes it possible to obtain satisfactory results even without this requirement. ${ }^{36}$ An alternative way of finding the wave function from the distribution of $\rho^{\text {stat }}$ is described at the end of this Section.

The rigidity of the multipolar pseudoatoms ${ }^{7}$ (i.e. the independence of the populations of the thermal parameters) gives rise to the possibility of separating the effects of chemical bonds from the thermal "spreading" of $\rho$ and of constructing the pseudostatic electron density $\rho^{\text {stat }}$. When such construction is entirely adequate, the results should be independent of the experimental temperature. So far it has not been possible to achieve this. ${ }^{37}$ A more detailed analysis of the influence of thermal motion on the distribution of $\rho$ has been described. ${ }^{38-40}$
According to certain investigators, the clearest form in which the results of a precision X-ray diffraction analysis can be represented is in terms of the electrostatic potential $\varphi$ and the deformation electrostatic potential $\delta \varphi .^{41}$ The application of this method to organic crystals is extremely fruitful in the analysis of the effects of the chemical bond, intermolecular interaction, and reactivity. These questions have been discussed in other reviews, ${ }^{42,43}$ where the principal formulae can be found.

We may note the latest studies in this field. A method for the subdivision of the crystal space into molecules with the aid of the electroneutrality principle has been proposed ${ }^{44}$ and applied to dicyanodiamide. The $\delta \varphi$ distribution maps for the $\mathrm{C}_{13} \mathrm{H}_{8} \mathrm{~N}_{2}$ molecule have made it possible ${ }^{45}$ to discover the weakest bond in the cyclopropane ring: whereas on other bonds the deformation potential $\rho \varphi$ has a negative minimum, on this bond it is close to zero. This agrees with the chemical properties; even at room temperature, the substance undergoes unimolecular isomerisation with dissociation of the given bond.

The study of phosphorylethanolamine $\mathrm{C}_{2} \mathrm{H}_{8} \mathrm{NO}_{4} \mathrm{P}{ }^{46}$ showed that the large (in absolute magnitude) negative potential in the region of the ester oxygen atom of the phosphate group is not saturated even on formation of a hydrogen bond by the other oxygen atom of the same group. The layer made up of such molecules serves as a model of a phospholipid membrane and near the surface has regions of positive and negative potential, which enables the molecules of the layer to form hydrogen bonds with both donors and acceptors. Such "amphoteric" behaviour was not predicted in quantumchemical calculations for an isolated molecule.

The study of the molecular complex of thiourea with parabanic acid $\mathrm{CH}_{4} \mathrm{~N}_{2} \mathrm{~S} . \mathrm{C}_{3} \mathrm{H}_{2} \mathrm{~N}_{2} \mathrm{O}_{3}{ }^{47}$ revealed saddle points in the $\varphi$ distribution of approximately equal height in the regions of the $\mathrm{N}-\mathrm{H}$...O and $\mathrm{N}-\mathrm{H}$...S hydrogen bonds formed by the molecules of this layer. It was concluded on this basis that the energies of the two bonds are similar despite the considerable difference between their lengths $(0.27 \AA)$. It has also been shown that the interaction of molecules of different layers does not take place as a result of charge transfer but involves short contacts between the $S$ atoms and the $\mathrm{C}-\mathrm{C}$ bonds: there is a broad saddle point in the distribution of $\varphi$ in this region.

The electrostatic potential of the molecule permits a correct inference about its intermolecular interactions only in those cases where interactions involving monopoles predominate in electrostatic forces (for example, on protonation). The "dipolar electrostatic potential", 48 which describes the optimum orientation and energy of a single dipole located at a certain point in the electron distribution (in particular on the van der Waals surface of the molecule) is more suitable for characterising the interaction with a polar uncharged molecule. Such maps show, for example, that the preferred direction along which a nucleophile approaches a formaldehyde molecule does not lie in the plane of this molecule. ${ }^{48}$

The force density method, ${ }^{49}$ consisting in the analysis of the distribution of the modulus of the electrostatic force density $f_{\mathrm{A}}(\mathbf{r})=$ $\rho(\mathbf{r}) /\left(\mathbf{r}-\mathbf{R}_{\mathrm{A}}\right)^{2}$, acting on the nucleus $A$, located at the point $\mathbf{R}_{\mathrm{A}}$, from the side of the electron density at the point $r$ and diminishing (or intensifying) the destabilising repulsion from other nuclei, is close to the electrostatic potential method. For example, the function $f_{N}(r)$ for the $N_{2}$ molecule has peaks at the centre of the bond (stabilisation) and on the line obtained by producing the bond to the rear at the given atom (destabilisation). In the $\mathrm{LiF}$ molecule, similar $f_{\mathrm{F}}(\mathrm{r})$ peaks have been displaced towards the $\mathrm{F}$ nucleus, while $f_{\mathrm{Li}}(\mathbf{r})$ has a single peak coinciding with the centre of the $F$ atom. Apart 
from the differences between covalent and ionic bonds, the force density method makes it possible to investigate the character of the chemical bond in complex polycyclic structures.

However, analysis of the $\varphi$ and $f$ distributions as well as the use of local (effective) electrostatic characteristics apparently solves only a limited range of specific problems. The most universal and most widely used means for the primary interpretation of the distribution of $\rho$ is provided by the deformation electron density $\delta \rho$ function, which is extremely sensitive to details of the electronic structure of molecules and crystals. The regions of positive values of $\delta \rho$ are those where electron density accumulates compared with the precrystal, consisting of non-bonded atoms. However, the absence of positive $\delta \rho$ maxima on the bond (and even the negative values of $\delta \rho)$, which sometimes occur in the case of bonds formed by electronegative atoms $(\mathrm{N}, \mathrm{O}, \mathrm{F}$, etc.), by no means imply the absence of a bond.

One of the causes of such anomalies consists in the fact that thermal motion "blurs" the $\delta \rho$ map. Thus the dynamic function $\delta \rho$ for crystals of $p$-nitropyridine $\mathrm{N}$-oxide $\mathrm{C}_{5} \mathrm{H}_{4} \mathrm{~N}_{2} \mathrm{O}_{2}{ }^{50}$ has low values

in the region of the $>\mathrm{N} \rightarrow \mathrm{O}$ bonds and the lone pairs of the $\mathrm{O}$ atom,

whereas considerable peaks appear in these regions on maps which are constructed with the aid of the multipolar resolution and afford a pseudostatic picture. Furthermore, the large angle procedure for the refinement of thermal parameters can lead to the smoothing of the anisotropy of the electron distribution. This effect causes a particularly marked reduction of the height of the LEP peaks, which are located closer to the nucleus than the peaks on the bonds. ${ }^{51}$

However, the type of bond has a decisive influence on the height of the $\delta \rho$ peak. The causes of the redistribution of electron density, arising from the preparation of the atoms of the premolecule for bonding (promotion of electrons, orientation, polarisation, and hybridisation of orbitals) and from the formation of bonds proper (interference of the orbitals and charge transfer) have been listed ${ }^{52}$ in terms of hybrid orbitals (HO).

We shall consider initially the effects due to the formation of bonds. For a bond between atoms 1 and 2, the bonding molecular orbital, made up of the singly populated HO $\psi_{1}$ and $\psi_{2}$, assumes the following form in terms of the LCAO MO approximation:

$$
\Psi=\left(1+2 \lambda S+\lambda^{2}\right)^{-1 / 2}\left(\psi_{1}+a_{\psi_{2}}\right),
$$

where $S$ is the overlap integral. The electron density of this orbital is

$$
\begin{aligned}
& \rho_{M O}=2 \Psi^{2}=\left(1+2 \lambda S+\lambda^{2}\right)^{-1}\left(2 \psi_{1}^{2}+2 \lambda^{2} \psi_{2}^{2}+4 \lambda \psi_{1} \psi_{2}\right)= \\
& =\left(\psi_{1}^{2}+\psi_{2}^{2}\right)+\left(1+2 \lambda S+\lambda^{2}\right)^{-1}\left(4 \lambda \psi_{1} \psi_{2}-2 \lambda S\left(\psi_{1}^{2}+\psi_{3}^{2}\right)\right)+ \\
& \quad+\left(1+2 \lambda S+\lambda^{2}\right)^{-1}\left(1-\lambda^{2}\right)\left(\psi_{1}^{2}-\psi_{2}^{2}\right)=\rho_{0}+\Delta \rho_{i}+\Delta \rho_{c t} .
\end{aligned}
$$

For HO with similar energies, $\lambda \simeq 1$ and $\Delta \rho_{c t} \simeq 0$. The deformation density is then $\Delta \rho_{i}$ and is approximately proportional to the overlap density $\psi_{1} \psi_{2}$ at the maximum. By analogy with the interaction of light waves, this effect is referred to as interference. With increase in bond length, the overlap density and hence $\Delta \rho_{i}$ diminish. Furthermore, for large bond lengths (especially between transition metal atoms), electron correlation, not taken into account in the MO method, makes an appreciable contribution to the decrease of $\Delta \rho_{i} .^{51}$

If the interacting $\mathrm{HO}$ are identical, then the quantity $\Delta \rho_{i}$ is a maximum at the centre of the bond. On the other hand, if they have similar energies but different extents in space, the greatest overlap density (and hence the $\Delta \rho_{i}$ maximum) should be attained closer to the centre of the more compact orbital. Even greater changes in elecron density are induced by differences between the orbital energies. The energy of the HO diminishes with increase in its electronegativity and $s$ character. If the energy of $\psi_{2}$ is greater than that of $\psi_{1}$, then $\lambda<1$ and the charge transfer effect $\Delta \rho_{c t} \simeq$ $\psi_{1}^{2}-\psi_{2}^{2}$ arises. Ultimately the $\Delta \rho$ peak shifts in the direction of the atom 1 .

We shall now consider a hypothetical distribution of electron density of the premolecule on transition of the atoms to the valence state (i. e. during the preparation of the atoms for the formation of a bond). According to the concept of hybridisation, when a $\mathrm{HO}$ is formed with participation of the atomic $s$ and $p$ orbitals, the populations $n_{s}$ and $n_{p}$ change (electrons are promoted from the $s$ sublevel to the $p$ sublevel). In particular, in the case of ideal $s p^{3}$-hybridisation of the $\mathrm{C}$ atom, $n_{s}=2, n_{p}=2 / 3$, and $n_{s}^{\prime}=$ $n_{p}^{\prime}=1 ;$ for the $\mathrm{O}$ atom, $n_{s}=2, n_{p}=3 / 4$, and $n_{s}^{\prime}=n_{p}^{\prime}=6 / 4$. This leads to some transfer of the electron density of a spherical atom from its centre to its periphery. The subsequent orientation of the $\mathrm{HO}$ is determined by the nearest neighbours of the atom in the premolecule and fixes both the position of the singly populated HO involved in the bonds and the doubly populated HO (i. e. lone pairs). After the orientation of the HO, the change in the population of the $p$ orbital directed along the bond line is

$$
\Delta n_{p}=3 /{ }_{4}+1 / 12\left(n_{2}+n_{3}+n_{4}\right)-n_{p},
$$

where the numerical coefficients correspond to the contributions of the $p$ orbital to the $s p^{3}$-HO with the populations $n_{i}$. For the carbon and oxygen atoms, $\Delta n_{p}=0.33$ and -0.17 , i. e. $\rho$ accumulates between carbon atoms and diminishes between oxygen atoms, while between oxygen and carbon atoms the electron density shifts from oxygen to carbon. Electrons always accumulate in the direction of the LEP.

$$
\Delta n_{p}^{\prime}=3 / 2+1 / 12\left(n_{2}+n_{3}+n_{4}\right)-n_{p} .
$$

However, the simple scheme described does not take into account certain fairly important effects. For example, under the influence of the environment the atomic orbitals undergo a change in shape (are polarised). In particular, the $1 s$ orbital of the $\mathrm{H}$ atom contracts slightly on formation of the bond. Furthermore, the differences between the electronegativities of the substituents or steric stresses can lead to deviation of the hybrid orbitals from the ideal $s p^{3}, s p^{2}$, and $s p$ orbitals. Finally, the populations of the interacting orbitals can differ from unity. Thus, in the $\mathrm{Mn}_{2}(\mathrm{CO})_{10}$ dimer examined below, the population of the $d$ orbitals forming the $\mathrm{Mn}-\mathrm{Mn}$ bond diminishes under the influence of the ligand field, which is in fact the main reason for the absence of the $\delta \rho$ peak on this bond. ${ }^{51}$ However, despite the above stipulations and effects due to the formation of bonds, the height of the $\delta \rho_{\max }$ peak usually varies in parallel with the quantity $n_{p}^{\prime}-n_{p}$ according to Angermund et al.; ${ }^{10}$ this indicates the predominance of the orientation effect.

In order to isolate the chemical bond effect in a pure form, the sum of the electron densities of the oriented atoms $\rho_{0}^{(o r)}$ was subtracted ${ }^{52,53}$ from the total function $\rho$; the resulting function was called the chemical deformation density $c \delta \rho=\rho-\rho_{0}^{(o r)}$.

When the functions $c \delta \rho$ are used, we in fact abandon a universal reference standard. The next step in this direction is taken when the "fragment-formation" density $f \delta \rho$ is introduced; ${ }^{54}$ this is obtained when the electron density of the fragments is subtracted from the total function $\rho$. The $f \delta \rho$ maps can be used to investigate the redistribution of electrons when the reacting species interact. 
In particular, in the study of coordination compounds, it is natural to differentiate the central atom and the ligands as the fragments. This was done in a quantum-chemical study of the distribution of $\rho$ in the $\mathrm{Co}_{2}(\mathrm{CO})_{8}$ molecule, ${ }^{54}$ which showed that the interaction of a $\mathrm{CO}$ molecule with a metal atom leads to the outflow of electron density from the $\mathrm{C}-\mathrm{O}$ axis into the region of the $p$ orbitals of oxygen and carbon perpendicular to this line. The function $f \delta \rho$ makes it possible to observe the redistribution of electrons on formation of a covalent bond between radicals or molecules. The peak due to the $\mathrm{Mn}-\mathrm{Mn}$ bond, which is absent from $\delta \rho$ maps, and also the displacement of the lone electron pairs of the oxygen atoms in the equatorial $\mathrm{CO}$ groups in the direction of the "foreign" $\mathrm{Mn}$ atom can be seen on the $f \delta \rho\left[\mathrm{Mn}_{2}(\mathrm{CO})_{10}-2 \mathrm{Mn}(\mathrm{CO})_{5}\right]$ map constructed ${ }^{54}$ from the results of non-empirical calculations, while on $\delta \rho$ maps the differences between the electron distributions of the axial and equatorial ligands are inappreciable.

It has been shown ${ }^{55}$ with the aid of $f \delta \rho$ that, on formation of the $\mathrm{H}_{3} \mathrm{~N} \rightarrow \mathrm{BH}_{3}$ donor-acceptor bond, an appreciable decrease in electron density is observed in the first place in the region of the $p_{\sigma}$ orbital of the $B$ atom, extended along this bond, with the simultaneous decrease in electron density on the corresponding orbital of the $\mathrm{N}$ atom and on the ammonia $\mathrm{H}$ atoms (the $\sigma$-donor effect of nitrogen) and in the second place there is a small transfer of electron density from the perpendicular $p_{\pi}$ orbital of the B atom to the orbital of the $N$ atom oriented in parallel (the $\pi$-acceptor effect).

The above examples of quantum-chemical calculations indicate the possibility of the effective employment of the $f \delta \rho$ function also for the primary interpretation of the experimental distributions of $\rho$.

With the aid of difference functions, it is possible to discover also the redistribution of electrons under the influence of external forces. Thus the change in electron density in the $\mathrm{H}_{2} \mathrm{O}$ molecule under the influence of an external homogeneous electrostatic field is demonstrated by "polarisation" deformation density $p \delta \rho$ maps. ${ }^{56}$ In order to discover the effects of the ionic environment in the $\mathrm{H}_{2} \mathrm{O}$ molecule and in the $\mathrm{HC}_{2} \mathrm{O}_{4}^{-}$ion, the distributions of the "double" deformation density $d \delta \rho=\delta \rho^{\prime}-\delta \rho$ were constructed, the $\delta \rho^{\prime}$ distributions being obtained by means of a non-empirical calculation modelling the influence of the crystal structure by point charges. ${ }^{57}$

In the study of intermolecular interactions (including hydrogen bonds and other specific contacts), the "molecular-deformation" density $m \delta \rho$ (the difference between the experimental function $\rho$ in the molecular crystal and the superposition of the molecular distributions of $\rho$ calculated for the experimental geometry of the molecules) may prove useful. The $m \delta \rho$ maps, constructed for formamide ${ }^{58}$ and oxalic acid, ${ }^{59}$ reflect the influence of intermolecular interactions, not only on the atoms involved in hydrogen bonds, but also on the inner parts of the molecule.

A fundamentally different and extremely promising procedure for the primary interpretation of the function $\rho$ (topological analysis) was developed by Bader and co-workers (see Refs. 60-62 and the bibliography quoted therein); this method provides one of the possible justifications of the qualitative concept of the classical theory of molecular structure. The principles of Bader's theory are described below and the results of the latest studies of his group as well as the applications of this approach to the experimental distributions of $\rho$ are examined.

The topological properties of the function $\rho(\mathbf{r})$ are revealed in the analysis of the vector field of the gradient $\nabla \rho(\mathbf{r})$. Here one uses a series of auxiliary concepts (they are illustrated by Fig. 1, where the vector field of the $\mathrm{CH}_{2}=\mathrm{CHF}$ molecule is presented as an example).
1. The surface $S$, at each point of which the vector flux of the gradient is zero [i.e. $\nabla \rho(\mathbf{r}) . n(\mathbf{r})=0$, where $n$ is the normal] is called the zero flux surface.

2. The curve such that the tangent to each point on it coincides with the direction of the gradient vector is referred to as the trajectory or gradient line. Each trajectory is one of the solutions of the equation $d \mathbf{r}(s) / d s=\nabla \rho[\mathbf{r}(s)]$ and is specified by the formula

$$
\mathbf{r}(s)=\mathbf{r}_{0}+\int_{0}^{s} \nabla \rho(\mathbf{r}(t)) d t .
$$

3. The point at which the gradient $\nabla \rho(r)$ becomes zero is called critical (the point $\mathbf{r}_{c}$ ). This point is characterised by the rank $\lambda$ and the symbol $\sigma$. The rank $\lambda$ is equal to the number of non-zero values of the curvature matrix (Hessian) with the elements

$$
H_{i j}=\left(\partial^{2} \rho / \partial \mathbf{r}_{i} \partial \mathbf{r}_{j}\right) \mathbf{r}=\mathbf{r}
$$

and the symbol $\sigma$ represents the difference between the number of positive and negative eigenvalues.
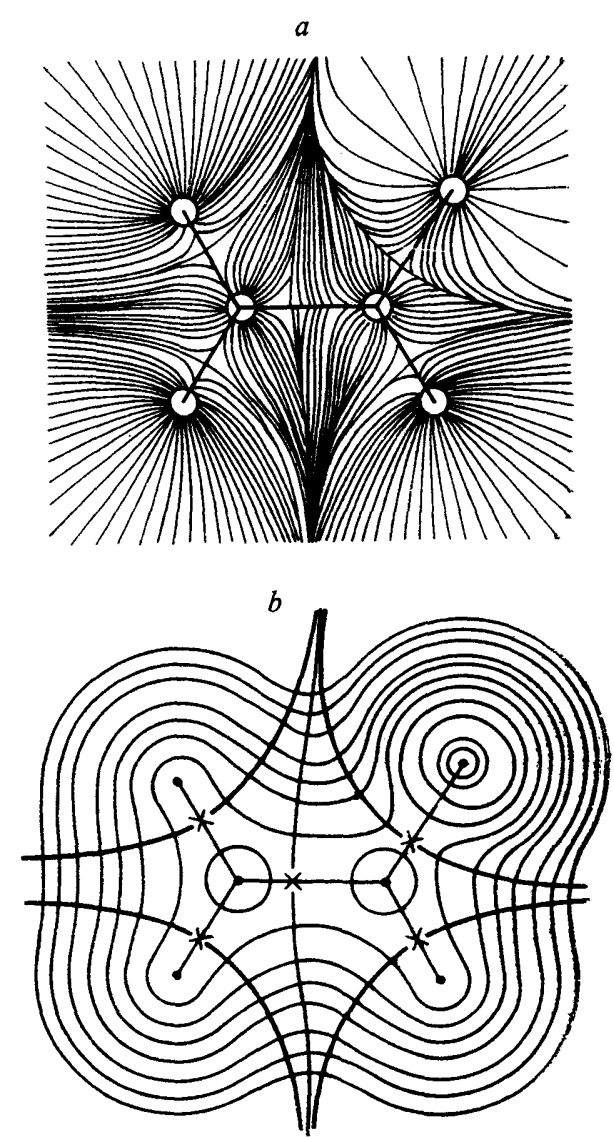

Figure 1. Topological analysis of the vector field of the $\mathbf{C H}_{2}=\mathrm{CHF}$ molecule (section coinciding with the plane of the molecule): (a) gradient lines terminating at the atomic nuclei, bond lines, and zero flux surfaces dividing the basins of the atoms are shown; (b) the critical points $(3,-3)$ and $(3,-1)$, the bond lines, and the zero flux surfaces against the background of the distribution of electron density are shown. 
The critical points $(\lambda, \sigma)$, for which $\lambda<3$, are degenerate (unstable): for any change in the function $\rho(\mathbf{r})$, induced by the shift of the nuclei, these points either vanish or break up into a certain number of non-degenerate (stable) critical points. Such points are encountered rarely but play an important role because they indicate the possibility of structural changes in the system.

Non-degenerate critical points (for which $\lambda=3$ ) can refer to one of four types: $(3,-3)$ (maxima), $(3,-1)$ and $(3,+1)$ (saddle points), $(3,+3)$ (minima).

The critical point of the type $(3,-3)$ represents a set of ends $(s \rightarrow \infty)$ of gradient trajectories which do not lie on the surfaces $S$; thus the role of "sinks" (attractors) of the vector field $\nabla \rho$ is attributed to these points.

The set of trajectories terminating at the given attractor forms the "basin" of this attractor. Two neighbouring basins are separated by the surface $S$, on which is located the critical point of the $(3,-1)$ type [crossing point (pass)]. The trajectories located on the surfaces $S$ terminate at the critical points $(3,-1)$; a pair of trajectories, terminating at two neighbouring attractors and forming the "bond line", arise from each such point. Along this line, the electron density $\rho$ is a maximum, i.e. small displacements perpendicular to this line lead to a decrease in $\rho$.

In Bader's theory, it is assumed that the minima in the function $\rho$ coincide with the atomic nuclei. + Nuclei are then connected by bond lines; the assembly of nuclei and bond lines forms a molecular graph. The atom is defined as a combination of the attractor (nucleus) and its basin.

Bader's theory also includes the analysis of the "structural stability" of molecular systems, whose description is outside the scope of the present review.

Various methods for detecting the location of atoms in a molecule have been described in the literature. ${ }^{64,65}$ However, the condition of the fulfillment of the principle of minimum action not only for the entire system (molecule) but also for its subsystems (atoms) requires that atoms be bounded by zero flux surfaces, as is done in Bader's theory. In this case, the atom in a molecule is described by the same equations of motion and theorems (for example, the virial theorem or the Ehrenfest theorem) as those applicable to the total system. Any property of an atom (charge, dipole moment, kinetic and potential energies) is calculated as the average value of a one-particle operator, which, however, is averaged only with respect to the volume of the atomic basin and not the entire space.

The topographical characteristics proposed by Bader also make it possible to describe important properties of interatomic bonds. The ratio of the bond length $l$ to the length of the internuclear vector and the distance from the corresponding critical point $(3,-1)$ to this vector serve as a measure of the curvature of the bond in molecules with steric strain or in non-equilibrium molecular systems. The degree of angular strain is measured as the difference between the angle between the bond lines and the angle between the bond axes.

$\dagger$ In a recent non-empirical study of free $\mathrm{Na}_{m}$ clusters $(m=2,4),{ }^{63}$ $\rho$ maxima were observed between nuclei, the $\mathrm{Na}$ atoms forming bonds only via these "pseudoatoms". Apparently this feature is characteristic of a metallic bond and the experimental observation of $\rho$ maxima at the centres of the vacancies in spherical packings is to be expected, for example, for crystals of alkali metals and beryllium.
The energy of the $A B$ bond is defined as

$$
E_{A B}=\alpha N_{A B} /\left(\mathbf{R}_{A}-\mathbf{R}_{B}\right)^{2},
$$

where $\alpha$ is a dimensionless coefficient (it is assumed that it is equal to 3 for the bonds in alkanes) and $N_{A B}$ is a measure of the number of bond electrons. The latter quantity is assumed to be zero for free atoms, while for the deformed atoms in the molecule

$$
N_{A B}=\left(\mathbf{R}_{A}-\mathbf{R}_{B}\right) \oint \rho(\mathbf{r}) \mathrm{n}_{A}(\mathbf{r}) d S_{A B},
$$

where $S_{A B}$ is the area of the zero flux surface separating two basins and $n_{A}(r)$ is the unit vector of the normal to $S_{\mathrm{AB}}$ directed from the atom $\mathbf{A}$ to the outside.

Important conclusions about the character of the bond can be reached from the analysis of the distribution of $\rho$ in the region of the critical point $(3,-1)$. By definition, the Hessian at the critical point has two negative $\left(\left|\lambda_{1}\right| \geqslant\left|\lambda_{2}\right|\right)$ and one positive $\left(\lambda_{3}\right)$ eigenvalue, each of which defines the curvature of $\rho$ along the corresponding eigenvector, i.e. at right angles to the bond $\left(\lambda_{1}\right.$ and $\left.\lambda_{2}\right)$ and along the bond $\left(\lambda_{3}\right)$. The multiplicity $n$ of the $\mathrm{C}-\mathrm{C}$ bond can be calculated from the electron density $\rho\left(\mathbf{r}_{c}\right)$ at this point. This is done by interpolating between the values of $\rho\left(\mathbf{r}_{c}\right)$ in the $\mathrm{C}_{2} \mathrm{H}_{6}, \mathrm{C}_{6} \mathrm{H}_{6}, \mathrm{C}_{2} \mathrm{H}_{4}$, and $\mathrm{C}_{2} \mathrm{H}_{2}$ molecules, for which $n=1,1.5,2$, and 3. The corresponding relation is expressed by the formula $n=\exp \left[6.458\left(\rho_{c}-0.252\right)\right]$.

The bond ellipticity $\varepsilon$ [where $\varepsilon=\left(\lambda_{1} / \lambda_{2}\right)-1$ ] is a measure of the deviation of the charge distribution along the bond trajectory from radial symmetry. This quantity is sensitive to the amount of " $\pi$ character" of the bond. If a single bond is conjugated with a double bond, then calculation for the former yields $n>1$ and $\varepsilon>0$.

However, the presence of the critical point $(3,-1)$ is only a necessary but not a sufficient condition for the existence of the covalent bonds between two atoms: such points exist in ion pairs, in molecular complexes with hydrogen bonds and charge transfer, and also in premolecules. The predominance in the internuclear region of the potential energy of the electrons (negative in sign) over the positive kinetic energy has been postulated as a sufficient condition. This empirical postulate has different mathematical expressions. Bader uses for this purpose the "charge concentration function": $g=-\nabla^{2} \rho$, where $\nabla^{2} \rho$ is the Laplacian of the electron density $\left(\nabla^{2} \rho=\right.$ $\left.\partial^{2} \rho / \partial x^{2}+\partial^{2} \rho / \partial y^{2}+\partial \rho / \partial z^{2}\right)$, expressing the overall curvature at the point with the coordinates $x, y$, and $z$ and equal, according to the virial theorem, to twice the sum of the kinetic and total energies of the electrons at this point. Other workers ${ }^{66}$ consider the total energy of the electrons (the sum of the kinetic and potential energies) at the critical point of the bond.

If $g\left(\mathbf{r}_{c}\right)>0$, then the quantity $\rho$ at the critical point exceeds its average value in a small region near this point, i.e. the charge accumulates in the internuclear space and the bond is regarded as covalent. The only exception is the $F_{2}$ molecule, which can be explained by the anomalously high intraatomic correlation of the electrons. For an ionic bond, $g\left(\mathbf{r}_{c}\right)<0$. Here the function $\rho$ exhibits a plateau in the plane perpendicular to the bond line and the positive values of $g$ are localised near the nuclei on either side of the plane. The same picture is observed for hydrogen bonds and van der Waals interactions.

More detailed topographical analysis of the function $g$ also yields useful results. In an isolated atom, the maximum values of $g$ are observed at the point corresponding to the nucleus and on $n-1$ concentric spheres, where $n$ is the principal quantum number. Since an evident analogy with the shell model of the atom is to be seen 
here, the outer sphere with an increased charge concentration is referred to as the valence shell. When the atom forms chemical bonds, the valence shell is distorted and loses the constancy of $g$ at each of its points; local $g$ maxima appear on it, i. e. two dimensional attractors $(2,-2)$ and between them saddle points $(2,0)$ appear on it. The trajectories $\nabla g$, originating from the saddle points, divide the valence shell into two-dimensional basins each of which contains one maximum. If $g>0$ at a given maximum, then a region of increased charge concentration, i.e. a region where $g>0$, corresponds to it (evidently such regions can overlap).

In Bader's view, analysis of maps of the distribution of the function $g$ provides a physical justification of the concept of electron pairs. Furthermore, the number, positions, and dimensions of the regions with an increased charge concentration agree with Gillespie's ideas ${ }^{67}$ of the repulsion of localised pairs, explain Pearson's theory of hard and soft acid and bases, ${ }^{68}$ and can serve as a basis for the determination of the direction of electrophilic and nucleophilic attack on the orientation of the reacting molecules at the start of the reaction. Bader believes that this approach is more correct than the electrostatic models usually employed nowadays, because molecules approaching one another "see not the charges on the atoms and not the distribution of the electrostatic potential but the system of local discharge and charge concentration centres described by the electron density Laplacian". The predictions of the intramolecular and intermolecular interactions, presupposing the mutual attraction of regions with different signs of $g$, have therefore proved to be extremely successful.

Thus the minimum in $g$ on the valence shell surface of the carbonyl carbon atom is located in the direction forming an angle of $110^{\circ}$ with the $\mathrm{C}=\mathrm{O}$ bond. Accordingly, in crystal structures with shortened contacts between oxygen and carbonyl carbon atoms the $\mathrm{O} . . \mathrm{C}=\mathrm{O}$ angle is usually close to this value.

When the molecule contains competing active centres, analysis of the function $g$ makes it possible to predict the preferred direction of the reaction. For this purpose, one uses the concept of the "hardness" of the active centre. 68 The interaction of "like with like", i.e. of a harder nucleophile with a harder electrophile and conversely, is found to be preferred. According to Bader, the hardness of the nucleophile (electrophile) increases with decrease in the distance between the maximum (minimum) and the nucleus and also with increase in the absolute magnitude and curvature of $g$ at the extremum.

The approach developed by Bader and co-workers has been used recently to interpret the results of non-empirical quantum-chemical calculations for an increasing range of molecular systems (hydrocarbons, carbonium ions, carbenes, complex compounds, "non-rigid" molecules, molecular dimers). With the aid of this approach, it is possible to investigate intermolecular and intramolecular hydrogen bonds and the effects of hyperconjugation and heteroaromaticity. It has been shown ${ }^{69}$ that the properties of atoms and bonds and the influence of substituents on the position of the critical point can be associated with simple orbital models. The fact that the electron distribution in the premolecule $\rho_{0}$ contains valuable chemical information is somewhat unexpected: in particular, it makes it possible to divide the molecule into pseudoatomic fragments. ${ }^{70}$

It was noted previously that the Bader approach is promising not only as regards the calculated theoretical distributions $\rho(\mathbf{r})$ but also as regards their experimental values. The first studies on these lines $^{71,72}$ confirmed this conclusion. The ethane molecule in a crystal was investigated on the basis of the topographical analysis of the experimental function $\rho$ taking into account the specific limitations of the experiment. ${ }^{71}$ In contrast to the free molecule, here one observes a non-zero ellipticity of the $\mathrm{C}-\mathrm{C}$ bond, which can be treated as partial double bond character due to the influence of the environment. However, this effect can be explained also by the imperfection of the method: the static distribution of $\rho$ in the molecule is regarded as the sum of the static function $\rho_{0}$ of the premolecule and the dynamic function $\delta \rho$, which precludes the complete exclusion of the influence of thermal motion. A $\nabla^{2} \rho$ map has been used ${ }^{72}$ to describe non-classical many-centre bonds in the pseudocubic $\mathrm{V}_{4} \mathrm{~S}_{4}$ cluster. The conclusion that the $\mathrm{V}-\mathrm{V}$ and $\mathrm{V}-\mathrm{S}$ bonds are covalent, reached on its basis, served as a significant complement to the $\delta \rho$ map.

Thus topographical analysis of the distribution of $\rho$ is a powerful instrument and reveals many hidden features of electron density. However, like any universal method, it does not enable one to discover fully the chemical individuality of the compound. Comparision of the distributions of $\rho$ for the given substance and its chemical analogues helps to view this individuality from different aspects.

The necessity for a quantititative comparison of two charge distributions arises also in other cases (comparison of theory and experiment, comparison of the results of two different calculations or two experimental studies, etc.). Such comparison can be carried out on three levels: (1) the integral level (the outcome is expressed by a single parameter); (2) the local level (the outcome is represented by a set of values); (3) the continuous level (the outcome of the comparison is a continuous function).

The Carbo index serves as an integral characteristic of molecular similarity. ${ }^{73}$ For isoelectronic molecules, it can be reduced to the generalised Polanski number:

$$
K_{12}=\frac{\int \rho_{1} \rho_{2} d V}{\left(\int \rho_{1}^{2} d V\right)^{1 / 2}\left(\int \rho_{2}^{2} d V\right)^{1 / 2}} \simeq \frac{N}{2} \operatorname{tr}\left(P_{1} P_{2}\right)
$$

where $N$ is the number of electrons and $P_{1}$ and $P_{2}$ are the density matrices of the molecules 1 and 2 formulated in terms of a single basis set. The quantity $K_{12}$ depends on the method used to superimpose the molecules. On the basis of the similarity index for the reactants and products, it is possible, for example, to predict the stereospecificity of pericyclic reactions. ${ }^{74}$

Without dwelling in detail on the local comparison of the distributions of $\rho$, we shall mention only that the discrete characteristics, listed at the beginning of this section, or the properties of atoms and bonds considered by Bader can serve as parameters of such comparison.

Continuous comparison yields most information but is usually carried out at a qualitaltive level involving the visual comparison of two $\delta \rho$ maps. For example, the differences between two crystallographically independent thiourea molecules (the reasons for the differences were not discovered $)^{21}$ and three forsterite crystals (the reason is the inadequate allowance for the extinction manifested in different ways $)^{12}$ were observed in precisely this way.

A more complete and objective result is provided by a quantitative continuous comparison involving the construction of difference density maps. However, even for small differences between geometrical parameters of the molecular systems 1 and 2 compared, such constructions become ambiguous. Even in the simplest case, involving the comparison of two $\mathrm{N}_{2}$ molecules with internuclear distances of 1.09 and $1.11 \AA$, one can superimpose either the centres of gravity or one pair of nuclei. In the former case, the 
difference density map ${ }^{75}$ shows the accumulation of $\rho$ between the nuclei as the latter approach one another, while in the second it shows the accumulation of $\rho$ in the postnuclear region and in both cases there is a pair of intense peaks (positive and negative) corresponding to the nuclear displacements. The "point" generalised-difference density $\Delta \rho^{\prime}$ makes it possible to avoid the latter false effect and at the same time to discover the first two. Such density was derived by Steiner: ${ }^{75}$

$$
\Delta \varphi^{\prime}(R)=\rho_{1}\left(f_{1}(r)\right)-\rho_{2}\left(f_{2}\left(r^{\prime}\right)\right) .
$$

where $\mathbf{r}$ and $\mathbf{r}^{\prime}$ are the coordinates of the points corresponding to one another in the molecules 1 and 2 and $\mathbf{R}=\mathbf{f}(\mathbf{r})$ is the function of the change of variables mapping the coordinates of the nuclei of the first molecule $\mathbf{r}_{i}$ on the ideally disposed reference points $\mathbf{R}_{i}$. The integral of $\rho$ with respect to the volume of the molecule, which is equal to the number of electrons, must be invariant relative to such change of variables:

$$
\int \rho(\mathbf{r}) d \mathbf{r}^{3}=\int \rho^{\prime \prime}(\mathbf{R}) d \mathbf{R}^{3}=\int \rho^{\prime \prime}(f(\mathbf{r}))\left|\frac{d^{3} \mathbf{R}}{d^{3} \mathbf{r}}\right| d \mathbf{r}^{3} .
$$

In order to take into account this factor, a "volume" generaliseddifference density $\Delta \rho$ " was constructed:

$$
\Delta \rho^{\prime \prime}(\mathbf{R})=\rho_{1}(\mathbf{r})\left|\frac{d^{3} \mathrm{f}_{1}(\mathbf{r})}{d^{3}}\right|-\rho_{2}(s)\left|\frac{d^{3} \mathrm{f}_{2}\left(\mathbf{r}^{\prime}\right)}{d^{\prime} \mathbf{r}^{\prime}}\right|,
$$

where $\left.\mid d^{3} \mathbf{f}_{1}(\mathbf{r}) / d^{3} \mathbf{r}\right\}$ is the Jacobi determinant.

In the case of the $N_{2}$ molecule, the distribution of $\Delta \rho$ " on approach of the nuclei proved to be similar to the distribution of $\delta \rho$ and was interpreted as partial transfer of electrons from the $p_{\pi}$ to the $p_{\sigma}$ orbital. A similar electron redistribution was noted also in the case of ethane on transition from the eclipsed to the hindered configuration. This effect is not manifested in the rigid rotation of methyl groups and has been observed only with the aid of generalised-difference maps. ${ }^{75}$ A significant disadvantage of the study described is an extremely artificial and ambiguous construction of the function $f$ by the method of segmental-linear interpolation along each coordinate of the Cartesian system. This led to breaks on the $\Delta \rho$ " maps and to a dependence of $\Delta \rho$ " on the choice of direction of the axis.

The coordinate transformation method was also used ${ }^{76}$ for the solution of the problem of recovering the wave function from precision XRDA data. The change of variables leaves the nuclei in place and distorts the electron density of the premolecule $\rho_{0}$ until it coincides fully with the experimental density:

$$
\rho(\mathbf{r})=\left|\frac{d^{3} \mathbf{f}}{d^{3} \mathbf{r}}\right| \rho_{0}(\mathbf{f}(\mathbf{r})) .
$$

The transformation $\mathbf{f}$ found from this condition is then applied to the wave function of the precrystal in order to obtain the wave function of the crystal. A local-scaling transformation, in which $\mathbf{r} \mid \mathbf{f}(\mathbf{r})$ is used as f. By applying the approach described to the problem of the construction of the generalised-difference density, one can ensure the uniqueness of the function of the change of variables by the requirement for the complete coincidence of the electron densities of the premolecules of the systems being compared.

Another procedure for the quantitative comparison of the charge distributions $w_{1}$ and $w_{2}$, where $w=\rho, \varphi, \delta \rho, c \delta \rho, \nabla^{2} \rho$, etc., which also ensures the uniqueness of the function $f$, has been proposed. ${ }^{77}$ In particular, it was noted that, in constructing $\mathbf{f}$, it is essential to take into account a series of factors dictated by the physical significance of the problem: the movement of the region near the nuclei without deformation, a strong dependence of the changes in electron density on the changes in the electrostatic potential and the smooth (non-oscillatory) character of these changes. Mathematically the uniqueness of the function $f$ is ensured by minimising a functional for which one can use the measure of the distortion of space $^{77}$ or the information entropy. In the comparison of the distributions of $\rho$ in systems 1 and 2 , the function $f$ can be conveniently represented as a homeomorphism mapping the trajectories $\nabla \rho_{1}$ onto the trajectories $\nabla \rho_{2}$. (For molecules or molecular fragments having equivalent structures according to Bader, ${ }^{60}$ such homeomorphism exists by definition). In the latter case it is reasonable to impose the requirement that the function $f$ should bring into coincidence not only the nuclei but also other critical points, as well as the zero flux surface and bond lines.

If a comparison is carried out in order to interpret chemical effects, it is essential to take into account the difference between the thermal vibrations in the systems being compared. Within the framework of the Debye-Waller approximation, ${ }^{7}$ this can be done in two ways: by constructing the pseudostatic densities $\rho^{\text {stat }}$ or by blurring $\rho_{2}$ by the thermal vibrations characteristic of system 1 (with the aid of a convolution transformation) within the framework of the multipolar pseudoatom or solid state models. The function $\Delta \rho "$ constructed reflects the influence of the environment and the substituents on the distribution of $\rho$ in the molecules. ${ }^{77}$

Thus the quantitative comparison of the charge distributions makes it possible (after the appropriate choice of the objects to be compared) to characterise comprehensively the chemical individuality of the molecules and to extract fundamentally new information from $\mathrm{X}$-ray diffraction data.

\section{Trends in the distributions of deformation electron density}

Hitherto deformation density maps have been constructed for more than 120 organic compounds. The list, which includes 96 organic substances investigated in this way, has been published in a review. ${ }^{6}$ Table 1 lists the organic substances investigated most recently (from 1983 to 1987); among them, one may encounter compounds mentioned in the review of Tsirel'son and Zorkii ${ }^{6}$ but reinvestigated recently. The most complex structural formulae of the compounds in Table 1 are shown in Fig. 2.

The available experimental data make it possible to note the following qualitative feature of the distributions of $\delta \rho$ :

(1) the presence of a peak on the covalent bond line (the exceptions are discussed below);

(2) dependence of the form of this peak on the $\pi$ character of the bond;

(3) the shift of the peak away from the bond axis in sterically strained systems;

(4) asymmetry of the peak of a polar bond;

(5) the shift of the peaks of electron-deficient bonds to the bisector of the bond angle (up to the fusion of these peaks);

(6) the presence of a single peak or two peaks in the region of lone electron pairs of the heteroatom;

(7) the slight deformation of the LEP peak on formation of weak and moderately strong hydrogen bonds;

(8) approximate symmetry of the strong hydrogen bond $\mathrm{X}-\mathrm{H}$...Y relative to the centre of the $X Y$ section. 
Table 1. The list of substances investigated.

\begin{tabular}{|c|c|c|c|c|}
\hline Empirical formula & Name of substance & Structural formula & Structural class & Refs. \\
\hline $\mathrm{CH}_{4} \mathrm{~N}_{2} \mathrm{O}^{*}$ & Urea & $\left(\mathrm{H}_{2} \mathrm{~N}\right)_{2} \mathrm{CO}$ & $P \overline{4}{ }_{1} m, Z=2(2 \mathrm{~mm})$ & [9] \\
\hline $\mathrm{CH}_{4} \mathrm{~N}_{2} \mathrm{~S}^{*}$ & Thiourea & $\left(\mathrm{H}_{2} \mathrm{~N}\right)_{2} \mathrm{CS}$ & Pnma, $Z=4\left(m^{2}\right)$ & {$[9,21]$} \\
\hline $\mathrm{CH}_{4} \mathrm{~N}_{2} \mathrm{~S} \cdot \mathrm{C}_{3} \mathrm{H}_{2} \mathrm{~N}_{2} \mathrm{O}_{3}$ & Thiourea-parabanic acid complex & $\left(\mathrm{H}_{2} \mathrm{~N}\right)_{2} \mathrm{CS} \cdot \mathrm{C}(\mathrm{O}) \mathrm{NHC}(\mathrm{O}) \mathrm{NHC}(\mathrm{O})$ & $P 2_{1} / m, Z=2(m, m)$ & {$[47]$} \\
\hline $\mathrm{C}_{2} \mathrm{H}_{2} \mathrm{O}_{4} \cdot 2 \mathrm{H}_{2} \mathrm{O}^{*}$ & $\alpha$-Oxalic acid dihydrate & $(\mathrm{COOH})_{2} \cdot 2 \mathrm{H}_{2} \mathrm{O}$ & $P 2 / c, Z=2(1 ; 1)$ & {$[11,59,78]$} \\
\hline $\mathrm{C}_{2} \mathrm{H}_{6} \mathrm{NO}_{2} \cdot \mathrm{Br}^{-}$ & Glycinium bromide & {$\left[\mathrm{H}_{3} \mathrm{NCH}_{2} \mathrm{COOH}\right]^{+} \mathrm{Br}-$} & & {$[79]$} \\
\hline $\mathrm{C}_{2} \mathrm{H}_{6} \mathrm{~N}_{2} \mathrm{O}_{2}$ & $N$-Methyl- $N^{\prime}$-methoxydiazene $N$-oxide & $\mu u c-\mathrm{CH}_{3} \mathrm{ON}=\mathrm{N}(\mathrm{O}) \mathrm{CH}_{3}$ & $P 2_{1} / c, Z=4(1)$ & {$[80]$} \\
\hline $\mathrm{C}_{2} \mathrm{H}_{8} \mathrm{TeCl}_{2}$ & Dimethyitellurium dichloride & $\left(\mathrm{CH}_{3}\right)_{2} \mathrm{TeCl}_{2}$ & $P 2_{1} / c, 2=4(1)$ & {$[81]$} \\
\hline $\mathrm{C}_{8} \mathrm{O}_{2} \mathrm{Cl}_{3}^{-} \cdot \mathrm{C}_{8} \mathrm{H}_{6} \mathrm{NO}^{+}$ & Hydroxypyridinium trichloroacetate & $\mathrm{CCl}_{3} \mathrm{COO}^{-}\left[\mathrm{HN}(\mathrm{CH})_{4} \mathrm{COH}\right]^{+}$ & not quoted & {$[82]$} \\
\hline $\mathrm{C}_{2} \mathrm{Cl}_{2} \mathrm{P}$ & Bis(trichloromethyl)trichlorophosphorane & $\left(\mathrm{CCl}_{9}\right)_{2} \mathrm{PCl}_{3}$ & $R 3 m, Z=6(3 m)$ & {$[83]$} \\
\hline $\mathrm{C}_{9} \mathrm{H}_{2} \mathrm{~N}_{2} \mathrm{O}_{2} \mathrm{~S}$ & 1,6-Dioxa-2,5-diaza-6a-thiopentalene & see Fig. 2 & not quoted & {$[84]$} \\
\hline $\mathrm{C}_{3} \mathrm{H}_{4} \mathrm{~N}_{2}$ & 2-Aminopropenonitrile & $\mathrm{CH}_{3}=\mathrm{C}(\mathrm{CN}) \mathrm{NH}_{2}$ & $P 2_{1} 2_{1} 2_{1}, Z=8(1)$ & {$[85]$} \\
\hline $\mathrm{C}_{9} \mathrm{H}_{9} \mathrm{~N}$ & Trimethylamine & $\left(\mathrm{CH}_{3}\right)_{3} \mathrm{~N}$ & $P \overline{3}, Z=2$ & {$[86]$} \\
\hline $\mathrm{C}_{4} \mathrm{H}_{2} \mathrm{~N}_{2} \mathrm{O}_{4}$ & $2,4,5,6(1 H, 3 H)$-Pyrimidinetetrone(alloxan) & $\mathrm{NHC}(\mathrm{O}) \mathrm{C}(\mathrm{O}) \mathrm{C}(\mathrm{O}) \mathrm{NHC}(\mathrm{O})$ & $P 4_{1} 2_{1} 2_{1}, Z=4(1)$ & {$[87]$} \\
\hline $\mathrm{C}_{5} \mathrm{H}_{8} \mathrm{NO}^{*}$ & 2-Pyridone & $\mathrm{NC}(\mathrm{OH})(\mathrm{CH})_{3} \mathrm{CH}$ & $P 2_{1} 2_{1} 2_{1}, Z=4(1)$ & ]88] \\
\hline $\mathrm{C}_{6} \mathrm{H}_{5} \mathrm{~N}_{2} \mathrm{Cl}$ & 2-Amino-5-chloropyridine & $\mathrm{H}_{2} \mathrm{NCHNCHC(Cl)CHCH}$ & $P 2_{1} / C, Z=4(1)$ & {$[89]$} \\
\hline $\mathrm{C}_{6} \mathrm{H}_{6} \mathrm{NO}^{+}$ & $\mathrm{Sec} \mathrm{C}_{2} \mathrm{O}_{2} \mathrm{Cl}_{3}$ & & & \\
\hline $\mathrm{C}_{6} \mathrm{H}_{10} \mathrm{O}_{6}$ & $\beta$-DL-arabinose & see Fig. 2 & $P 2 / c, Z=4(1)$ & {$[15]$} \\
\hline $\mathrm{C}_{4} \mathrm{H}_{2} \mathrm{~N}_{4}$ & 1,1,2,2-Ethanetetracarbonitrile & $(\mathrm{CN})_{2} \mathrm{CHCH}(\mathrm{CN})_{2}$ & $P 2_{1} / c, Z=2(1)$ & {$[91]$} \\
\hline $\mathrm{C}_{8} \mathrm{H}_{7} \mathrm{~N}$ & 4Mahylpyridine & $\mathrm{CHCHNCHCHCHCH}$ & $I 4_{1} / a, Z=8(2)$ & {$[92]$} \\
\hline $\mathrm{C}_{8} \mathrm{H}_{8} \mathrm{~F}_{4}$ & 1,1,4,4-Tetrafluorocyclohexane & $\mathrm{CF}_{2} \mathrm{CH}_{2} \mathrm{CH}_{2} \mathrm{CF}_{2} \mathrm{CH}_{2} \mathrm{CH}_{2}$ & Pnnm, $Z=2(2 / m)$ & {$[30,93]$} \\
\hline $\mathrm{C}_{8} \mathrm{H}_{12} \mathrm{~N}_{4} \mathrm{O}_{4}$ & $\begin{array}{l}\text { 4,5.10.11-Tetraosa-1,2,7,8-tetraaza tricyclo- } \\
{\left[6.4 .1 .1^{2.7}\right] \text { tetradecane }}\end{array}$ & see Fig. 2 & $P \overrightarrow{1}, Z=1(\overrightarrow{1})$ & {$[95]$} \\
\hline $\mathrm{C}_{8} \mathrm{D}_{2} \mathrm{O}_{4} \mathrm{~F}_{4}^{*}$ & Perdeuteriotetrafluoroterephthalic acid & $\mathrm{C}_{6} \mathrm{~F}_{4}(\mathrm{COOD})_{2}$ & $P \overline{1}, Z=1(\overline{1})$ & {$[97]$} \\
\hline $\mathrm{C}_{6} \mathrm{~N}_{2} \mathrm{~F}_{4} *$ & Tetrafluoroterephthalonitrile & $n-\mathrm{C}_{6} \mathrm{~F}_{4}(\mathrm{CN})_{2}$ & $\mathrm{Cmca} ; Z=4(2 / m)$ & {$[30,98]$} \\
\hline $\mathrm{C}_{10} \mathrm{H}_{9} \mathrm{~N}_{2} \mathrm{OSCl} *$ & 2-(2-Chlorobenzoimino)-1,3-thiazolidine & see Fig. 2 & $P 2_{1} / c ; Z=4(1)$ & {$[84,94]$} \\
\hline $\mathrm{C}_{10} \mathrm{H}_{10} \mathrm{~N}_{2} \mathrm{O}_{2}^{*}$ & 2,5-Bis(ethyleneimino)-1,4-benzoquinone & $\mathrm{CH}_{2} \mathrm{CH}_{2} \mathrm{~N}-\mathrm{C}$ & $P \overline{1}, Z=1(\overline{1})$ & {$[99-101]$} \\
\hline $\mathrm{C}_{10} \mathrm{H}_{12} \mathrm{Se}_{4}^{1 / 2+} \cdot \frac{1}{2} \mathrm{AsF}_{6}$ & $\begin{array}{l}\text { Bistetramethyitetraselenofulvalene } \\
\text { hexafluoroarsenate }\end{array}$ & see Fig. 2 & not quoted & {$[96]$} \\
\hline $\mathrm{C}_{12} \mathrm{H}_{12} \mathrm{~N}_{2} \mathrm{O}_{2} \mathrm{~S}^{*}$ & 3-Benzimino-4-methyl-1,2,4-0xathiazine & see Fig. 2 & $P 2 / c, Z=4(1)$ & {$[84,94]$} \\
\hline $\mathrm{C}_{12} \mathrm{H}_{11} \mathrm{~N}_{2} \mathrm{SiCl}_{3}$ & $\begin{array}{l}\text { 1-(Trichorosilyl)-1,2,3,4-tetrahydro- } \\
\text { 1,10-phenanthroline }\end{array}$ & see Fig. 2 & $P 2_{1} / m, Z=2(m)$ & {$[102]$} \\
\hline $\mathrm{C}_{13} \mathrm{H}_{8} \mathrm{~N}_{2}$ & $\begin{array}{l}\text { Tricyclo[4.4.1. } 0^{1.9} \text { undeca-2,4,7,9-tetraene- } \\
\text { 11,11-dicarbonitrile }\end{array}$ & see Fig. 2 & $P 2_{1} 2_{1} 2_{1}, Z=4(1)$ & {$[45]$} \\
\hline $\mathrm{C}_{18} \mathrm{H}_{18}{ }^{*}$ & 9-t-Butylanthracene & & $P 21 / c, Z=4(1)\}$ & {$[10,103$} \\
\hline $\mathrm{C}_{18} \mathrm{H}_{18}$ & 9-t-Butyl-9,10-Dewar-anthracenc & & $\left.P 2_{1} / c, 2=4(1)\right\}$ & $104,105]$ \\
\hline $\mathrm{C}_{19} \mathrm{H}_{22} \mathrm{NO}_{4}^{+} \cdot \mathrm{Cl}^{-} \cdot 2 \mathrm{H}_{2} \mathrm{O}$ & Naloxone hydrochloride dihydrate & $\sec \mathrm{Fi}_{\mathrm{g}} .2$ & not quoted & {$[31]$} \\
\hline $\mathrm{C}_{20} \mathrm{H}_{28} \cdot 0,86 \mathrm{Ar}$ & Tetra-t-butyltetrahedrane & see Fig. 2 & $P 6_{3} / m, Z=6(\overline{1})$ & {$[106]$} \\
\hline $\mathrm{C}_{20} \mathrm{H}_{36} *$ & Tetra-t-butylcyclobutadiene & $\mathrm{C}_{4}(\text { mpem } \cdot \mathrm{Bu})_{4}$ & $C 2 / c, 2=4(1)$ & {$[107]$} \\
\hline $\mathrm{C}_{2 \mathrm{~A}} \mathrm{H}_{20} \mathrm{Si}$ & Tetraphenylsilicon & $\left(\mathrm{C}_{6} \mathrm{H}_{5}\right)_{4} \mathrm{Si}$ & $P \overline{4} 2_{1} c, Z=2(\overline{4})$ & {$[108]$} \\
\hline $\mathrm{C}_{2 \mathrm{~g}} \mathrm{H}_{2 \mathrm{~g}} \mathrm{P}_{2}$ & 1,1-diphosphinoethylene & $\mathrm{H}_{2} \mathrm{C}=\mathrm{C}\left(\mathrm{PPh}_{2}\right)_{2}$ & not quoted & [109] \\
\hline & 1,1-Diphosphinoethylene dioxide & $\mathrm{H}_{2} \mathrm{C}=\mathrm{C}\left(\mathrm{POPh}_{2}\right)_{2}$ & not quoted & [109] \\
\hline
\end{tabular}

*Compounds already mentioned in an earlier review ${ }^{6}$ but reinvestigated.

The height of the covalent bond peak

In general, the height of the $\delta \rho_{\max }$ peak is not an unambiguous characteristic of the bond strength. Firstly (as a consequence of the lack of standard methods), the differences between the experimental conditions and the refinement procedures lead to a dependence of the results on the method in which it was obtained. ${ }^{6}$ Secondly, the thermal vibrations of the molecules and atoms in different structures can "blur" the $\delta \rho$ peaks to different extents, while the static distributions of $\delta \rho$ depend on temperature and the model used as a consequence of the insufficiently accurate allowance for thermal 


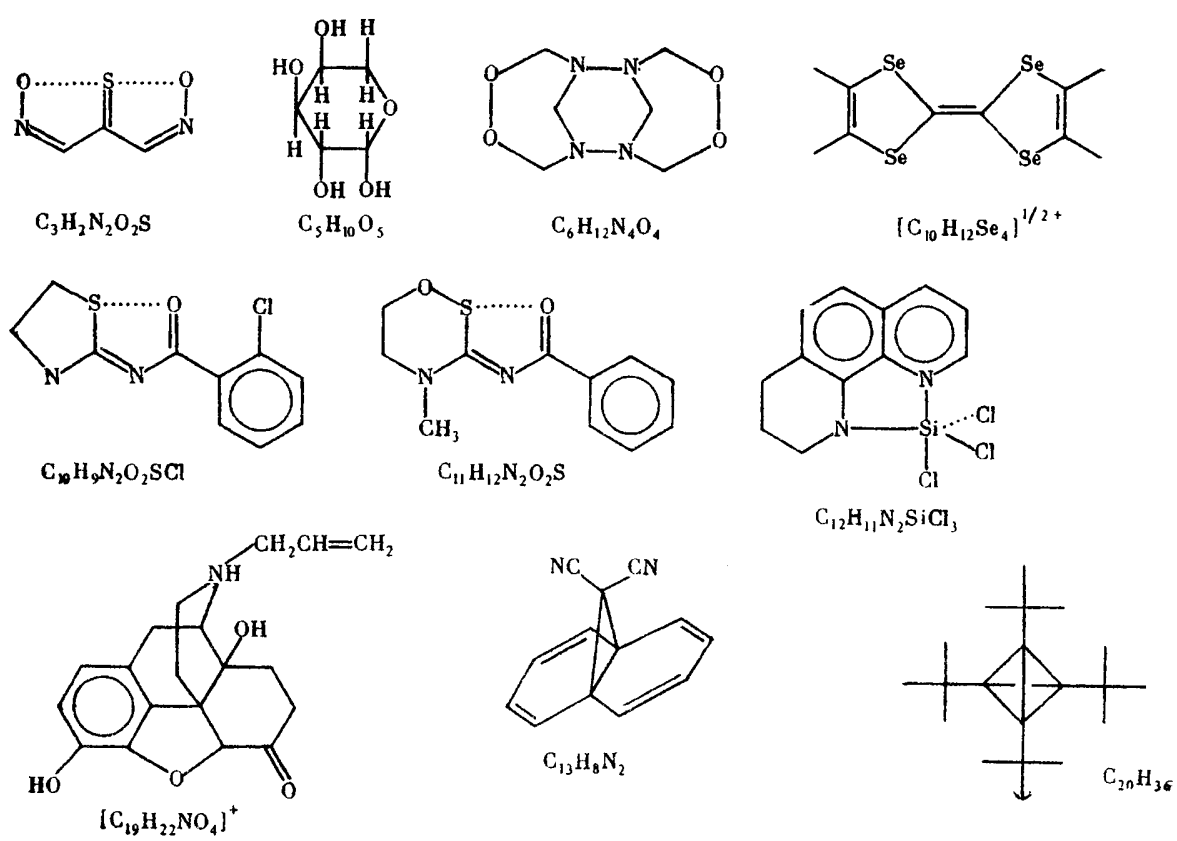

Figure 2. Structural formulae in Table 1.

motion. $^{21,37}$ Thirdly, as regards bond strength, the comparison of the deformation densities on bonds of this type (for example, $\mathrm{O}-\mathrm{O}$ and $\mathrm{N}-\mathrm{N}$ ) is known a priori to be incorrect. ${ }^{87}$

However, the differences between the values of $\delta \rho_{\max }$ in a single molecule can indicate the non-equivalence of isotypical bonds, which has been confirmed both theoretically and experimentally. For example, in the case of 9-t-butylanthracene $\mathrm{C}_{18} \mathrm{H}_{18}$, the values of $\delta \rho_{\max }$ are approximately proportional to the overlap populations ${ }^{10}$ calculated with the aid of the extended Hückel method. The extremely small value of $\delta \rho_{\max }$ on the elongated $\mathrm{C}-\mathrm{C}$ bridge bond in the compound $\mathrm{C}_{13} \mathrm{H}_{8} \mathrm{~N}_{2}{ }^{45}$ indicates that this is the weakest bond in the molecule. This conclusion is fully confirmed by chemical properties.

In certain cases, $\delta \rho_{\max }$ proved to be smaller than the background level; one then speaks of the absence of a peak on the bond. The bond between inverted carbon atoms in the propellane derivative $\mathrm{C}_{17} \mathrm{H}_{19} \mathrm{~N}$, having the normal length, has been examined as an example in a review. ${ }^{6}$ Theory describes such a bond with the aid of $s p^{4}$-hybrid [sic] orbitals whose overlap is indeed small. ${ }^{110}$

The causes of the systematic absence of peaks on bonds of the $\mathrm{C}-\mathrm{F}$ and $\mathrm{O}-\mathrm{O}$ types as well as certain others ${ }^{7}$ were indicated above in Section III. The splitting of the peak on bonds formed by a silicon atom $\left(\mathrm{Si}-\mathrm{Si},{ }^{26} \mathrm{Si}-\mathrm{Cl},{ }^{102}\right.$ and $\left.\mathrm{Si}-\mathrm{C}^{108}\right)$ is sometimes also observed.

\section{The form of the covalent bond peak}

Single, double, and triple bonds differ, apart from the values of $\delta \rho_{\max }$, by the form of the equal deformation density surfaces. In the ideal case, single and triple bonds are manifested as peaks having the shape of ellipsoids of rotation (the axis of rotation is extended along the bond axis) with different ratios of the longitudinal and transverse half-axes. ${ }^{11}$ The peak of the double bond in ethylene is extended at right angles to the plane of the molecule. ${ }^{112}$ When single and multiple bonds alternate in the molecule, conjugation arises: the density of $\pi$-electrons is displaced from the multiple to the single bond (the mesomeric effect is designated in the structural formula by a curved arrow). This is reflected in the shape of the deformation density peaks.

A characteristic example is provided by tetrafluoroterephthalodinitrile $\mathrm{C}_{8} \mathrm{~N}_{2} \mathrm{~F}_{4}:^{30}$<smiles>N#Cc1c(F)c(F)c([Ge]2CCCC2)c(F)c1F</smiles>

The form of the peaks due to different bonds on the $0.1 \mathrm{e} \AA^{-3}$ multipolar pseudostatic $\delta \rho$ distribution surface is characterised by the following values (in $\AA$ ).

$\begin{array}{lcccc}\text { Maximum width in the molecular } & C\left(1^{\prime}\right)-C(1) & C(1)-C(2) & C(2)-C(3) & C(3)-\mathrm{N} \\ \begin{array}{l}\text { plane }\left(d_{1}\right) \\ \text { Maximum width at right angles to }\end{array} & 0.89 & 0.89 & 0.93 & 1.30 \\ \begin{array}{l}\text { the plane }\left(d_{2}\right) \\ \text { Bond length }\end{array} & 1.41 & 1.28 & 1.02 & 1.23 \\ & 1.38 & 1.40 & 1.43 & 1.15\end{array}$

The sizes of the peaks confirm the existing ideas about the mesomeric effect and show that the $\pi$ character of the bonds diminishes monotonically in the sequence $\mathrm{C}\left(1^{\prime}\right)-\mathrm{C}(1), \mathrm{C}(1)-\mathrm{C}(2)$, $\mathrm{C}(2)-\mathrm{C}(3)$, since the quantity $d_{2} / d_{1}$ diminishes (the latter is confirmed also by the increase in bond length). This means that the structure of the six-membered ring approaches the quinonoid structure, while the linear $\mathrm{C}(2) \mathrm{C}(3) \mathrm{N}$ fragment approaches the 
cumulene type. We may note that the dynamic $\delta \rho$ distribution does not exhibit the quantitative features described. The existence of the inductive effect (transfer of electron density from $\mathrm{C}$ to $\mathrm{F}$ atoms and from the $\mathrm{C}$ atom to the $\mathrm{N}$ atom) follows from the atomic charges: F $0.13 \mathrm{e}$; $C(1) 0.13 \mathrm{e}$; $\mathrm{C}(2) 0.05 \mathrm{e}$; $\mathrm{C}(3) 0.11 \mathrm{e}$; $\mathrm{N} 0.15 \mathrm{e}$.

The alternation of charges in a chain of four carbon atoms, which agrees with the ideas about the inductive effect, has been discovered also in the molecule of barbital $\mathrm{C}_{8} \mathrm{H}_{12} \mathrm{~N}_{2} \mathrm{O}_{3}{ }^{113}$

Polar bonds exhibit asymmetry in the $\delta \rho$ peak relative to the centre of the $\mathrm{A}-\mathrm{X}$ internuclear section. For single bonds, having no $\pi$-component, the following regular feature is observed. If the bond polarity, measured by the quantity $\Delta q_{\mathrm{AX}}=q_{\mathrm{A}}-q_{\mathrm{X}}$ (where $q$ is the atomic charge) is large (of the order of $1 \mathrm{e}$ ), the $\delta \rho$ maximum is displaced towards the nucleus of the electronegative atom $X$, the function $\delta \rho$ diminishing faster in the direction of the $X$ nucleus than in the opposite direction. $\neq$ In organic molecules, purely single and at the same time polar bonds are encountered rarely; there are virtually no such bonds among organic substances for which carefully constructed $\delta \rho$ maps exist, (only in this case is there any point in discussing such fine details). Therefore examples of the features described can be more easily found among inorganic substances (the $\mathrm{B}-\mathrm{N}$ bond in the cubic $\mathrm{BN},{ }^{115}$ the $\mathrm{Si}-\mathrm{O}$ bond in coesite, ${ }^{116}$ and the $\mathrm{Te}-\mathrm{Cl}$ bond in $\mathrm{C}_{2} \mathrm{H}_{2} \mathrm{TeCl}_{2}{ }^{81}$ ). Weakly polar bonds (for example, $\mathrm{N}-\mathrm{O}, \mathrm{C}-\mathrm{N}$, and $\mathrm{C}-\mathrm{O}$ in $\mathrm{C}_{6} \mathrm{H}_{12} \mathrm{~N}_{4} \mathrm{O}_{4}{ }^{95}$ ) do not exhibit such asymmetry.

The characteristic features of bonds with a definite contribution of the $\pi$-component can be clearly seen in relation to barbital $\mathrm{C}_{8} \mathrm{H}_{12} \mathrm{~N}_{2} \mathrm{O}_{3} .{ }^{116}$ A shift of the charge on the $\mathrm{C}-\mathrm{N}$ and $\mathrm{C}-\mathrm{O}$ bonds in the $\pi$-region towards $\mathrm{N}$ and $\mathrm{O}$ bonds and in the plane of the molecule towards $\mathrm{C}$ bonds is observed in this instance. In the $\pi$-region, a similar feature, confirmed by theoretical calculations, is shown also by the carbonyl group in the urea $\mathrm{CH}_{4} \mathrm{~N}_{2} \mathrm{O}$ molecule. Such behaviour of the $\pi$-charge can be explained by the schematic illustration of the mesomeric shift of electrons:

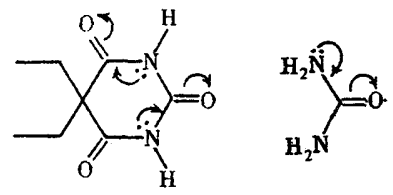

Hence it is clear that the population of the $p_{\pi}$ orbitals of the $N$ and $O$ atoms is greater than unity and that of the $C$ atom does not exceed unity. The differences between the orbital populations induce the asymmetry of the $\delta \rho$ peaks in the $\pi$-region. As regards the $\sigma$-charge, the example of parabanic acid $\mathrm{C}_{3} \mathrm{H}_{2} \mathrm{~N}_{2} \mathrm{O}_{3},{ }^{47}$ two investigations of which led to completely different forms of the $\delta \rho$ peak in the $\sigma$-region of the $\mathrm{C}-\mathrm{N}$ bond, makes it necessary to exercise caution in drawing inferences about the shape of the multiple bond peak in the molecular plane.

The asymmetry of the $\delta \rho$ peak for non-equivalent $\mathrm{N}-\mathrm{Si}$ bonds in the compound $\mathrm{C}_{12} \mathrm{H}_{11} \mathrm{~N}_{2} \mathrm{SiCl}_{3}{ }^{102}$ with a trigonal-bipyramidal coordination of the $\mathrm{Si}$ atom is manifested in different ways (Fig. 3). On the $\mathrm{N}_{e}-\mathrm{Si}$ bond with an equatorially coordinated nitrogen atom,

$\ddagger$ We may note that the shift of the symmetrical peak to one of the nuclei can be induced not [only] by the bond polarity but by the difference between the sizes of the principal atomic regions (an example is provided by the $\mathrm{C}-\mathrm{S}$ bond in the thiazolidine ring $^{114}$ ). an appreciable displacement of the $\sigma$-density towards the $\mathrm{Si}$ atom and of the $\pi$-density towards the $N_{e}$ atom is observed. The long bond with the axially coordinated nitrogen atom $\left(\mathrm{N}_{a} \rightarrow \mathrm{Si}\right)$, which is formed by a donor-acceptor mechanism, exhibits different features. Its $\sigma$-component is displaced from the centre to the $\mathrm{N}_{a}$ atom and the $\pi$-component is virtually absent. Similarly to the single bonds discussed above, the quantity $\delta \rho$ diminishes in this instance from the maximum towards the $\mathrm{N}_{a}$ atom much more steeply than in the opposite direction.

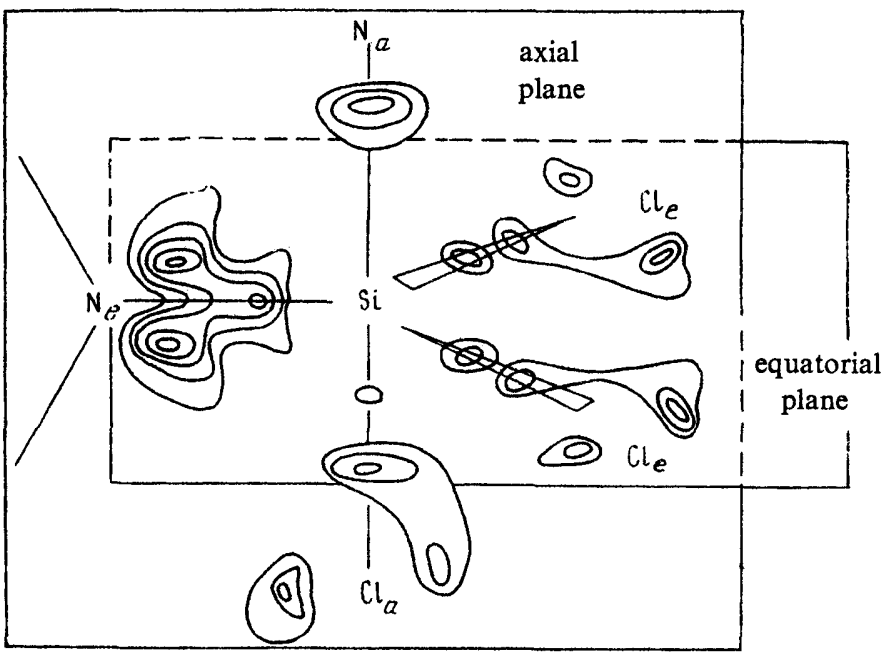

Figure 3. Schematic representation of the distribution of the deformation electron density in the $\mathrm{SiN}_{2} \mathrm{Cl}_{3}$ system.

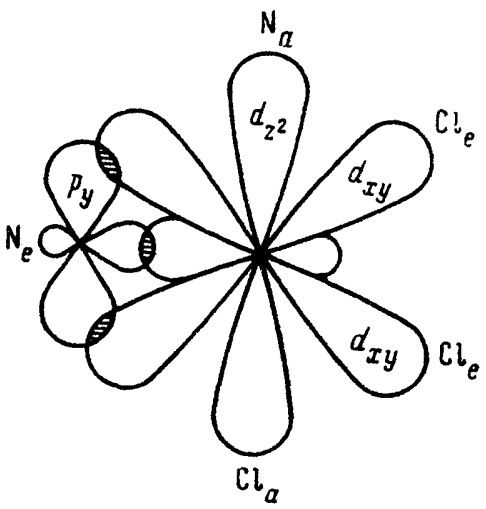

Figure 4. Interpretation of the electronic structure of the $\mathrm{SiN}_{2} \mathrm{Cl}_{3}$ system with the aid of atomic orbitals.

These facts can be explained with the aid of simple orbital ideas (Fig. 4). In the equatorial plane, the $\mathrm{Si}$ atom forms with the aid of $s p^{2}$-hybrid orbitals three $\sigma$-bonds (with the $\mathrm{N}_{e}$ atom and two $\mathrm{Cl}$ atoms). The $\mathrm{N}_{e}$ atom donates the electron pair occupying the $p_{y}$ 
orbital to the unoccupied $d_{x y}$ orbital of the $\mathrm{Si}$ atom and forms a $\pi$-bond. The bond with the axial $\mathrm{Cl}$ atom is produced by the $p_{z}$ orbital of the Si atom and the LEP of the $\mathrm{N}_{a}$ atom is transferred to the unoccupied $d_{z^{2}}$ orbital. The extent of the atomic $3 d$ orbitals in space explains the large distances from the $\mathrm{Si}$ nucleus to the peaks of the donor-acceptor bonds.

We may note that non-equivalence of the axial bonds is observed also in the trigonal-bipyramidal phosphorus compound $\mathrm{C}_{2} \mathrm{Cl}_{9} \mathrm{P}{ }^{83}$

\section{The position of the covalent bond peak}

In sterically strained systems, the $\delta \rho$ peaks deviate significantly from the bond lines in order to compensate for the decrease in the bond angle compared with the angle between the hybrid orbitals. ${ }^{6}$

The $\delta \rho$ map in the plane of the cyclopropane ring has been incorporated in textbooks and has become the classical image of banana bonds. ${ }^{117}$ Another example is the cyclobutadiene fragment of the $\mathrm{C}_{22} \mathrm{H}_{32} \mathrm{~S}_{2}$ molecule, in which the following features of the $\delta \rho$ distributions have been observed. ${ }^{118}$ In the plane passing at right angles through the centre of the $\mathrm{C}=\mathrm{C}$ bond, there is in this instance a two-humped peak and the saddle section of the peak, corresponding to the $\sigma$-component, is further away from the ring axis than the maxima corresponding to $\pi$-electrons; for a single bond, $\delta \rho$ is close to zero at the analogously located points (at the same distances from the plane of the ring where the double bond gives rise to $\delta \rho$ maxima). The observed picture can be explained as a result of the superposition of distributions characteristic of a bent $\sigma$-bond and the usual $\pi$-bond, but non-empirical quantum-chemical calculation, performed for cyclobutadiene, did not lead to the observation of the double-humped peak described.

In compounds with electron-deficient bonds, a trend has been noted towards a shift of the $\delta \rho$ peaks into the inner region of the angle between such bonds. ${ }^{119}$ Under these conditions the peaks fuse or a bridge is formed between them. Apart from inorganic compounds (metals, oxides) such features are observed in carbaboranes $^{120}$ and other boron compounds. Theory describes these compounds with the aid of three-centre two-electron orbitals localised at the centre of a three-membered ring made up of boron atoms.

\section{Peaks in the region of unshared electron pairs}

The height and shape of such peaks depend on the type of atom, its valence state, and the immediate environment. ${ }^{6}$ Non-valence interactions or conjugation with $\pi$-bonds usually greatly influence the LEP peaks.

The N(III) atom has a single LEP. The corresponding $\delta \rho$ peak is manifested most clearly in the case of $s p$-hybridisation in the $-\mathrm{C} \equiv \mathrm{N}$ : group (for example, in $\mathrm{C}_{8} \mathrm{~N}_{2} \mathrm{~F}_{4}{ }^{30}$ ). The LEP of the angular dicoordinate nitrogen, occupying an $s p^{2}$-hybrid orbital, is also clearly manifested, for example, in the $\mathrm{C}=\ddot{\mathrm{N}}-\mathrm{C}$ group (4-methylpyridine $\mathrm{C}_{6} \mathrm{H}_{7} \mathrm{~N} \quad{ }^{92}$ ) and the $\mathrm{C}=\ddot{\mathrm{N}}-\mathrm{O}$ group $\left(\mathrm{C}_{2} \mathrm{H}_{6} \mathrm{~N}_{2} \mathrm{O}_{2}{ }^{80}\right)$. However, in the case of nitrogen with a planar ternary coordination, where the LEP occupies a $p$ orbital, the maximum in the non-valence region is absent or is weak (in diformylhydrazine, ${ }^{121}$ acetamide, ${ }^{122}$ etc.). This is associated with the delocalisation of electrons on conjugation with double bonds. In the case of pyramidal coordination (in the compounds $\mathrm{C}_{6} \mathrm{H}_{12} \mathrm{~N}_{4} \mathrm{O}_{4} 95$ and $\mathrm{C}_{3} \mathrm{H}_{9} \mathrm{~N}{ }^{86}$ ), a diffuse LEP peak, occupying an $s p^{3}$ hybrid orbital, is observed near the $\mathrm{N}$ atom.
The two LEP of the oxygen atom give rise to two peaks located at an angle of $\sim 130^{\circ}$ in the case of $s p^{2}$-hybridisation. For $s p^{3}$-hybridisation, these LEP are usually manifested as a single longish peak (which agrees with non-empirical calculations for the $\mathrm{H}_{2} \mathrm{O}$ molecule), but the intermolecular interactions sometimes lead to the splitting of this peak into two components. Numerous examples have been discussed in a review. ${ }^{6}$ Here we shall only note that the peak corresponding to a single LEP can also be split, as has been observed for $\mathrm{TeO}_{2}{ }^{123}$

The remaining heteroatoms have been less thoroughly investigated. The unshared electron pairs of sulphur(II) usually behave like the oxygen LEP, but sometimes the height of their peak is comparable to the error of the determination of the $\delta \rho .^{6}$ In the pyramidal $\mathrm{SO}_{3}^{2-}$ ion, the single LEP of sulphur(IV) has been observed at the apex of the pyramid. ${ }^{124}$ The LEP peaks of the halogen atoms $X(X=F, C l)$ are manifested by a torus, whose axis coincides with $\mathrm{A}-\mathrm{X}$ bond line and whose centre is displaced towards the $A$ atom. ${ }^{83,94,102}$ More rarely they appear in the form of three lobes directed at right angles to this axis. ${ }^{89}$ Furthermore, there are negative minima in the direction opposite to the $\mathrm{A}-\mathrm{X}$ bond line indicating the participation of the $p_{\sigma}$ orbital of the atom $\mathrm{X}$ in the formation of this bond. A minimum is also observed on the $\mathrm{C}-\mathrm{F}$ bond itself. ${ }^{30,97}$

\section{Characteristic features of $\delta \rho$ in the region of hydrogen bonds}

It was noted previously ${ }^{6}$ that the $\delta \rho$ distribution along strong hydrogen bonds is approximately symmetrical relative to the midpoint despite the differences between $\mathrm{O}-\mathrm{H}$ and $\mathrm{O}$...H distances, while in the case of weak and moderately strong hydrogen bonds the $\delta \rho$ distributions are close to the superposition of $\delta \rho$ fragments.

However, in a more detailed analysis of strong $\mathrm{N}-\mathrm{H}$...N interactions, it was noted ${ }^{102}$ that the $\delta \rho$ distributions along the $\mathrm{N}$...H bond and along the covalent $\mathrm{N}-\mathrm{H}$ bond ${ }^{89}$ differ somewhat. In the former case, the $\delta \rho$ peak is polarised (it descends gently) in the direction of the $\mathbf{H}$ atom, while in the latter case it is polarised in the direction of the $\mathrm{N}$ atom. It has also been noted that the steepness of the peak of the LEP not involved in the intermolecular interaction is approximately the same on the side of the nucleus and on the opposite side. The polarisation of this peak indicates a weak hydrogen bond. The specific $\mathrm{C}-\mathrm{H}$...N interactions in the 1,2,3-triazine crystal were in fact discovered on the basis of this feature $^{10}$ (a priori the appearance of such hydrogen bond was regarded as unlikely).

The redistribution of $\delta \rho$ on formation of hydrogen bonds has been frequently studied theoretically. Comparison of non-empirical calculations for the $\mathrm{H}_{2} \mathrm{O}$ molecule in a free state and in a cluster, modelling the unit cell, indicates ${ }^{125}$ an increase in the polarity of the $\mathrm{O}-\mathrm{H}$ bonds and the transfer of electron density from the LEP and the hydrogen atoms to the $O$ atom. On the other hand, the presence of short contacts (the formation of strong hydrogen bonds) increases the LEP peak. In the presence of several nearest neighbours, the changes in $\delta \rho$ are superimposed. For this reason, an increase in the number of neighbours blurs the characteristic features of the $\delta \rho$ distribution.

Thus according to the data presented, the idea that the $\delta \rho$ in weak and moderately strong hydrogen bonds is the sum of the deformation density of the LEP and the covalently bound hydrogen appears to be fairly approximate. 


\section{Redistributions of $\delta \rho$ induced by specific non-valence interactions}

As an example of the influence of non-valence interactions on $\delta \rho$, we shall present the results of a study ${ }^{94}$ in which the intramolecular<smiles>[X][SH](C)OC=C(C)C</smiles>

interactions in the $\mathrm{C}_{10} \mathrm{H}_{9} \mathrm{~N}_{2} \mathrm{OSCl}$ and $\mathrm{C}_{11} \mathrm{H}_{12} \mathrm{~N}_{2} \mathrm{O}_{2} \mathrm{~S}$ molecules were compared (the lengths of the S...O contacts are 2.68 and $2.52 \AA$ respectively). In the former case, two peaks of the LEP of the carbonyl $\mathrm{O}$ atom can be seen in the $\delta \rho$ distribution, while the peaks near the $\mathrm{S}$ atom are absent. In the latter case, the peaks due to the LEP of oxygen have almost completely vanished, while the $S$ atom is surrounded by an intense $\delta \rho$ halo. Model calculations carried out with the aid of the extended Hückel method have shown that the order (covalence) of the bond between the atoms involved in these contacts reaches 4 and $25 \%$ respectively (compared with the covalent $\mathrm{S}-\mathrm{O}$ bond). $\S$ This can be explained by the fact that the interaction of the LEP of the $O$ atom and of the antibonding molecular orbital of the $\mathrm{S}-\mathrm{X}$ bond intensifies with increase in the electronegativity of the atom $X$.

Yet another example of specific non-valence interactions is provided by the anomeric effect in $\beta$-DL-arabinose, ${ }^{15}$ which has been detected in the molecular fragment

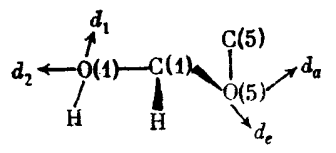

where the letters $d$ denote the LEP. It was established that the heights of the $\delta \rho$ peaks, corresponding to the unshared electron pairs $d_{a}, d_{e}$ and $d_{2}$ are similar. For the $d_{1}$ pair, this quantity is smaller, because this LEP interacts with the $C(1)-O(5)$ bond. It has also been noted that the $\delta \rho$ peaks on the "anomeric" $C(5)-O(5)$ and $\mathrm{O}(1)-\mathrm{H}$ bonds are significantly lower than on other bonds.

Manifestations of intermolecular specific non-valence interactions have been observed ${ }^{81}$ in $\left(\mathrm{CH}_{3}\right)_{2} \mathrm{TeCl}_{2}$ crystals. Here the orbital of one molecule, occupied by the unshared pair of the $\mathrm{Cl}$ atom, interacts with the antibonding orbital of the $\mathrm{Te}-\mathrm{C}$ bond of another molecule, which increases the level of $\delta \rho$ on the Te...Cl section.

From the standpoint of $\delta \rho$ distributions, the non-specific intermolecular interactions in organic crystals have so far been scarcely investigated. The effects associated with them are found at the limits of accuracy of the precision X-ray diffraction analysis but non-empirical quantum-chemical study of the intermolecular forces appears even more complex. It is therefore possible that new results, of fundamental importance for the theory of the structure of molecular condensed phases, will in fact be obtained in this precise region.

\section{References}

1. A Hartman, E F Hirshfeld Acta Crystallogr., 1966, 20(1) 80-82

2. P Coppens Science, 1967, 158 1577-1579

ๆNon-empirical calculations for similar systems ${ }^{126}$ yield similar results $(10-30 \%$ covalence) only when the $d$ orbitals of the $S$ atom are included in the basis set.
3. R F Stewart J. Chem. Phys., 1968, 48(11) $4882-4889$

4. A M O'Connell, A I M Rae, E N Maslen Acta Crystallogr., 1966, 21(3) 208-219

5. C J Fritchie Acta Crystallogr., 1966, 20(1) 27-36

6. V G Tsirel'son, P M Zorkii Kristallokhimiya (Crystal Chemistry), Izd. VINITI, Moscow, 1986, Vol. 20, pp. 124-173

[Itogi Nauki i Tekhniki (Advances in Science and Engineering)]

7. V G Tsirel'son Kristallokhimiya (Crystal Chemistry), Izd. VINITI, Moscow, 1986, Vol.20, pp. 3-123 [Itogi Nauki $i$ Tekhniki (Advances in Science and Engineering)]

8. P M Zorkii Simmetriya Molekul i Kristallicheskikh Struktur (Symmetry of Molecules and Crystal Structures), Izd. Mosk. Gos. Univ., Moscow, 1986, 232 pp

9. D Mullen Acta Crystallogr., Sect. B, 1982, 38(10) 2620-2625

10. V K Angermund, $\mathrm{K} \mathrm{H}$ Claus, R Goddard, C Kruger Angew. Chem., 1985, 97(4) 241-252

11. J Dam, S Harkema, D Feil Acta Crystallogr., Sect. B, 1983, 39(6) $760-768$

12. R J Van der Wal, A Vos, A Kirfel Acta Crystallogr., Sect. B, 1987, 43(2) $132-143$

13. F S Nielsen, P Lee, P Coppens Acta Crystallogr., Sect. B, 1986, 42(4) $359-364$

14. F K Larsen, N K Hansen Acta Crystallogr., Sect. B, 1984, 40(3) $169-179$

15. F Longschambon, $\mathrm{H}$ Gillier-Pandraud, $\mathrm{R}$ Wiest, et al. Acta Crystallogr., Sect. B, 1985, 41(1) 47-56

16. W Wei J. Appl. Crystallogr., 1985, 18(6) 442-445

17. D M Collins, M C Mahar Acta Crystallogr., Sect. A, 1983, 39(2) $252-256$

18. E A Kuz'min, E A Soldatov, V A Lebedev, et al. Kristallografiya i Kristallokhimiya (Crystallography and Crystal Chemistry), Izd. Nauka, Moscow, 1986, pp. 87-94

19. B M Craven, H P Weber Acta Crystallogr., Sect. B, 1983, 39(6) $743-748$

20. U Drück, A Kutoglu Z. Kristallogr., 1984, 166(3-4) 233-244

21. A Kutoglu, C Scheringer, $\mathrm{H}$ Meyer, A Schweig Acta Crystallogr., Sect. B, 1982, 38(10) 2626-2632

22. C Frichberg Boron-Rich Solids, International Conference, New York, 1986, pp. 136-141

23. L Sawyer Biochim. Soc. Trans., 1986, 14(3) 535-538

24. A V Ivashin, I D Feranchuk Kristallografiya, 1989, 34(1) $39-46$

25. U Pietsch, V G Tsirelson, R P Ozerov Phys. Status Solidi, B, 1986, 137(2) $441-447$

26. M A Spackman Acta Crystallogr., Sect. A, 1986, 42(2) 271-281

27. C Scheringer, A Kutoglu Acta Crystallogr., Sect. A, 1983, 39(6) $889-891$

28. G A Ivanov-Smolenskii XIV Vsesoyuznoe Soveshchanie po Primeneniyu Rentgenovskikh Luchei $k$ Issledovaniyu Materialov: Tezisy Dokladov. Kishinev (The XIVth All-Union Conference on the Application of X-Rays to the Study of Materials: Abstracts of Reports. Kishinev), Kishinev, 1985, pp. 39, 40

29. B M Craven, R O Fox Jr, H-P Weber Acta Crystallogr., Sect. B, 1982, 38(7) $1942-1952$

30. F L Hirshfeld Acta Crystallogr., Sect. B, 1984, 40(5) 484-492

31. C L Klien, R J Mayeste, E D Stevens J. Am. Chem. Soc., 1987, 109(22) $6675-6681$

32. P Coppens Coord. Chem. Rev., 1985, 65(3) 285-307

33. C Lecomte, $\mathrm{R}$ H Blessing, P Coppens, A Tabard J. Am. Chem. Soc., 1986, 108(22) 6942-6950 
34. B N Figgis, P A Reynolds J. Chem. Soc., Dalton Trans., 1986, (1) $125-134$

35. O V Gritsenko, G M Zhidomirov Dokl. Akad. Nauk SSSR, 1987, 293(5) 1162-1166

36. J E Harriman Phys. Rev. A, Gen. Phys., 1986, 34(1) 29-39

37. K Hermansson, J O Thomas, I Olovsson Acta Crystallogr., Sect. C, 1984, 40(3) 335-340

38. K B Wiberg, J J Wendoloski J. Phys. Chem., 1984, 88(3) $586-593$

39. A Tachibana, T Yamabe, K Hori, V Asai Chem. Phys. Lett., 1984, 106(1-2) 36-40

40. A Tachibana, K Hori, Y Asai, T Yamabe J. Chem. Phys., 1984, 80(12) 6170-6178

41. D Schwarzenbach Chimia, 1983, 37(10) 373-378

42. R F Stewart Godishn. Yugosl. Cent. Kristallogr., 1982, 17 170-176

43. A A Varnek,R P Ozerov Elektronnye Sostoyaniya Molekul $i$ Khimicheskaya Svyaz' (The Electronic States of Molecules and the Chemical Bonds), Tr. Mosk. Khim.-Tekhnol. Inst., 1984, No. 134 , pp. $54-77$

44. M V Krasheninnikov VII Vsesoyuznyi Simpozium po Mezhmolekulyarnomu Vzaimodeistviyu i Konformatsiyam Molekul: Tezisy Dokladov. Pushchino (The VIIth All-Union Symposium on Intermolecular Interaction and Molecular Conformations: Abstracts of Reports. Pushchino), Pushchino, 1986, pp. 16,17

45. R Bianchi, T Pilali, M Simonetta Helv. Chim. Acta, 1984, 67(8) $1707-1712$

46. S Swaminathan, B M Craven Acta Crystallogr., Sect. B, 1984, 40(3) $511-518$

47. H-P Weber, B M Craven Acta Crystallogr., Sect. B, 1987, 43(1) 202-209

48. A Kumar, P C Mishra Proc. Indian Acad. Sci., Chem. Sci., 1987, 99(1-2) 113-118

49. L A Gribov, M M Raikhshtat Zh. Strukt. Khim., 1988, 29(6) $32-41$

50. N K Hansen, P Copens Acta Crystallogr., Sect. A, 1978, 34(4) 909-921

51. B Hall Chem. Scripta, 1986, 26(3) 389-394

52. K L Kunze, M B Hall J. Am. Chem. Soc., 1987, 109(25) 7617-7623

53. W H E Schwarz, L Mensching, P Valtazanos, W Von Niessen Int. J. Quantum Chem., 1986, 30(3) 439-444

54. W Heijser, E J Baerends, P Ros Faraday Discuss. Symp., 1980, $14211-234$

55. M B Hall Electron Distributions and the Chemical Bond (Edited by P Coppens, M B Hall), Plenum Press, New York, 1982, pp. $205-220$

56. M P C M Krijn, D Feil J. Phys. Chem., 1987, 91(3) 540-544

57. S Lunell J. Chem. Phys., 1984, 18(12) 6185-6193

58. E D Stevens, J Rys, P Coppens J. Am. Chem. Soc., 1978, 100(10) 2324-2329

59. D Feil Chem. Scripta, 1986, 26(3) 395-408

60. R F W Bader Chemical Applications of Topology and the Theory of Graphs [tentative transcription from Russian] (Translated into Russian), Izd. Mir, Moscow, 1987, pp. 54-71

61. R F W Bader, P J MacDougall J. Am. Chem. Soc., 1985, 107(24) $6788-6795$

62. K B Wiberg, R F W Bader, C D H Lau J. Am. Chem. Soc., $1987,109(4) 985-1012$
63. W L Cao, C Gatti, P J MacDougall, R F W Bader Chem. Phys. Lett., 1987, 141(5) 380-385

64. V M Tatevskii Teoriya Fiziko-khimicheskikh Svoistv Molekul $i$ Veshchestv (Theory of the Physicochemical Properties of Molecules and Substances), Izd. Mosk. Gos. Univ., Moscow, 1987, $239 \mathrm{pp}$

65. L Li, R G Parr J. Chem. Phys., 1986, 83(3) 1704-1711

66. D Cremer, E Kraka Croat. Chim. Acta, 1987, 57(5) 1259-1267

67. R J Gillespie Molecular Geometry (Translated into Russian), Izd. Mir, Moscow, 1975, 278 pp

68. R G Pearson J. Am. Chem. Soc., 1963, 85(22) 3533-3539

69. T S Slee J. Am. Chem. Soc., 1986, 108(24) 7541-7543

70. M A Spackman, E N Maslen J. Phys. Chem., 1986, 90(10) 2020-2027

71. P Kapphan, V G Tsirel'son, R P Ozerov V Vsesoyuznoe Soveshchanie po Organicheskoi Kristallokhimii. Chernogolovka (The Vth All-Union Conference on Organic Crystal Chemistry. Chernogolovka), Chernogolovka, 1987, p. 171

72. M Yu Antipin, Yu L Slovokhotov, Yu T Struchkov Dokl. Akad. Nauk SSSR, 1987, 296(5) 1143-1147

73. R Carbo, L Leyda, M Arnau Int. J. Quant. Chem., 1980, 17(5) 1185- 1189

74. R Ponec Collect. Czech. Chem. Commun., 1987, 52(3) 555-562

75. E Steiner Theor. Chim. Acta, 1982, 60(4) $561-572$

76. I Zh. Petkov, M V Stoitsov, E S Kryachko Ukr. Fiz. Zh., 1986, 31(5) $777-782$

77. A E Masunov, P M Zorkii Vestn. Mosk. Univ., Ser. 2; Khim., 1988, 29(2) $131-136$

78. V Wang, C J Tsai, W L Liu, L D Calvert Acta Crystallogr., Sect. B, 1985, 41(2) $131-135$

79. A Oscarsson Acta Crystallogr., Sect. B, 1976, 32(11) 2163-2170

80. G A Marchenko, L F Chertanova, M Yu Antipin, et al. Dokl. Akad. Nauk SSSR, 1987, 294(5) 1169-1172

81. R F Ziolo, J M Troup J. Am. Chem. Soc., 1983, 105(2) $229-235$

82. K Eichhorn Z. Kristallogr., 1983, 162(1-4) 67-68

83. M Yu Antipin, A N Chernega, Yu T Struchkov Kristallografiya, 1987, 32(3) 631-637

84. C Cohen-Addad, P Becker, M S Lehmann, et al. 9th Eur. Cryst. Meet: Abstr., Torino, 1985, Vol. 1, pp. 254-255

85. P Seiler, J D Dunitz Helv. Chim. Acta, 1985, 68(8) 2093-2099

86. A J Blake, E A V Ebsworth, A J Welch Acta Crystallogr., Sect. C, 1984, 40(2) 413-415

87. S Swaminathan, B M Craven, R K McMuller Acta Crystallogr., Sect. B, 1985, 41(1) 113-122

88. U Ohms, H Guth, E Hellner, H Dannöhl, A Schweig Z. Kristallogr., 1984, 169(1-4) 185-200

89. A Kvick, R Thomas, T F Koetzle Acta Crystallogr., Sect. B, 1976, 32(2) 224-231

90. J N Low, P Tollin, D W Young Acta Crystallogr., Sect. C, 1986, 42(4) $1045-1047$

91. J P Declercq, B Tinant, A Parfonry, et al. Acta Crystallogr., Sect., 1983, 39(10) $1401-1405$

92. U Ohms, H Guth, W Treutmann, et al. J. Chem. Phys., 1985, 83(1) 273-279

93. J D Dunitz, W B Schweizer, P Seiler Helv. Chim. Acta, 1983, 66(13) $134-137$

94. C Cohen-Addad, M S Lehmann, P Becker, et al. J. Chem. Soc., Perkin Trans. 2, 1984, 2(2) 191-196 
95. J D Dunitz, P Seiler J. Am. Chem. Soc., 1983, 105(14) 7056-7058

96. F Wudl, D Nalewajek, J M Troup, M W Extine Science, 1983, 222(4622) 415-417

97. G Blockus Kristallogr., 1982, 159(1-4) 19

98. F L Hirshfeld Acta Crystallogr., Sect. B, 1984, 40(6) 613-615

99. A N Chekhlov, S P Ionov Zh. Fiz. Khim., 1982, 56(11) 2656 [English Translation in Russ. J. Phys. Chem., 1982(11)]

100. A N Chekhlov, S P Ionov Zh. Fiz. Khim., 1983, 57(1) 131 [English Translation in Russ. J. Phys. Chem., 1983(1)]

101. A N Chekhlov, S P Ionov Zh. Fiz. Khim., 1983, 57(9) 2251 [English Translation in Russ. J. Phys. Chem., 1983(9)]

102. G Klebe, J W Bats, H Fuess J. Am. Chem. Soc., 1984, 106(18) 5202-5208

103. B Jahn, H Dreeskamp Ber. Bunsenges. Phys. Chem., 1984, 88(1) $42-46$

104. H Dreeskamp, B Jahn, J Pabst Z. Naturforsch., A, 1981, 36 $665-669$

105. K Angermund, R Goddard, C Krüger Acta Crystallogr., Sect. A, 1984, 40, Supplement, 06-2-16

106. H Irngartinger, R Jahn, G Maier, R Emrich Angew. Chem., 1987, 99(4) 356-357

107. H Irngartinger, M Nixdorf Angew. Chem., 1988, 121(7) $679-683$

108. V Gruhnert, A Kirfel, G Will, et al. Z. Kristallogr., 1983, 163(1-2) $53-60$

109. C Krüger, H Schmidbauer, G Müller 12th International Conference on Organometal. Chemistry: Abstracts, Vienna, 1985, Sect. 1, p. 398

110. M D Newton, J M Schulman J. Am. Chem. Soc., 1972, 94(3) $773-778$

111. R B Helmholdt, A F J Ruysink, H Reynaers, G Kemper Acta Crystallogr., Sect. B, 1982, 28(2) 318-319

112. G J H van Nes, A Vos Acta Crystallogr., Sect. B, 1979, 35(11) $2593-2601$

113. B M Craven, R O Fox, J H-P Weber Acta Crystallogr., Sect. B, 1982, 38(7) 1942-1952

114. J Kamo, N Tanaka, Y Matsuura, et al. Bull. Chem. Soc. Jpn., 1979, 52(3) 706-710

115. V V Chernyshev, G V Fetisov, L A Aslanov Novye Vozmozhnosti Difraktsionnykh, Rentgenospektral'nykh $i$ Elektrono-Mikroskopicheskikh Metodov Issledovaniya: Tezisy Dokladov (New Possibilities in Diffraction, X-Ray Spectroscopic, and Electron Microscope Research Methods: Abstracts of Reports), Moscow, 1987, p. 79

116. K L Geisinger, M A Spackman, G V Gibbs J. Phys. Chem., 1987, 91(12) $3237-3244$

117. J N Murrell, S Kettle, J Tedder The Chemical Bond (Wiley, New York, 1978) (Translated into Russian), Izd. Mir, Moscow, $1980,382 \mathrm{pp}$

118. H Irngartinger, H-L Hase, $\mathrm{K}-\mathrm{W}$ Schulte, A Schweig Angew. Chem., 1977, 89(1) $194-196$

119. V G Tsirel'son, Yu Z Nozik, R P Ozerov, V S Urusov Kristallokhimiya (Crystal Chemistry), Izd. VINITI, Moscow, 1986, Vol. 20, pp. 174-259 [Itogi Nauki i Tekhniki (Advances in Science and Engineering)]

120. M Yu Antipin, A V Polyakov, V V Grushin, Yu T Struchkov Z. Kristallogr., 1988, 185(1) 193-199

121. H Hope, T Ottersen Acta Crystallogr., Sect. B, 1979, 35(2) $370-372$
122. T Ottersen, J Almlöf, H Hope Acta Crystallogr., Sect. B, 1980, 36(6) 1147-1154

123. I I Kondratyuk, L L Muradyan, Yu V Pisarskii, V I Simonov Kristallografiya, 1987, 32(3) 609-617

124. J W Bats, H Fuess, Y Elerman Acta Crystallogr., Sect. B, 1986, 42(3) $552-557$

125. K Hermansson Acta Crystallogr., Sect. B, 1985, 41(3) $161-169$

126. J G Angyen, R A Poirier, A Kucsman, I G Csizmadia J. Am. Chem. Soc., 1987, 109(8) 2237-2245 